\title{
RECONSTRUCCIÓN PALEOAMBIENTAL DEL ÚLTIMO CICLO GLACIAL-INTERGLACIAL EN LA IBERIA CONTINENTAL: LA SECUENCIA DEL CAÑIZAR DE VILLARQUEMADO (TERUEL)
}

\author{
P. GONZÁLEZ-SAMPÉRIZ1* ${ }^{*}$ E. GARCÍA-PRIETO', \\ J. ARANBARRI' ${ }^{1}$, B.L. VALERO-GARCÉS ${ }^{1}$, A. MORENO ${ }^{1}$, \\ G. GIL-ROMERA ${ }^{1}$, M. SEVILLA-CALLEJO ${ }^{1}$, L. SANTOS ${ }^{2}$, \\ M. MORELLÓN ${ }^{3}$, P. MATA $^{4}$, A. ANDRADE ${ }^{5}$, J.S. CARRIÓN ${ }^{6}$ \\ ${ }^{1}$ Instituto Pirenaico de Ecología, CSIC, Av. Montañana 1005, 50059 Zaragoza. \\ ${ }^{2}$ Facultad de Ciencias, Universidad de A Coruña, Campus da Zapateira, 15071, Coruña. \\ ${ }^{3}$ Department of Surface Waters Eawag (Das Wasserforschungs-Institut des ETH-Bereichs), \\ Überlandstrasse 133. P. O. Box 611, 8600 Dübendorf, Suiza. \\ ${ }^{4}$ Instituto Geológico y Minero de España (IGME), C/ La Calera 1, 28760 Tres Cantos, Madrid. \\ ${ }^{5}$ Dpto. Geografía y Geología, Unidad Docente de Geología, Edificio de Ciencias, \\ Carretera Nacional II, km. 33600, 28871 Alcalá de Henares. \\ ${ }^{6}$ Dpto. Biología Vegetal. Fac. de Biología, Campus de Espinardo, \\ Universidad de Murcia, 30100 Murcia.
}

RESUMEN. La secuencia de El Cañizar de Villarquemado (NE Península Ibérica) constituye un registro excepcional que cubre los últimos ca. 130000 años de historia ambiental. Los análisis multidisciplinares que se han realizado hasta el momento incluyen un análisis palinológico preliminar y otros indicadores sedimentológicos, como susceptibilidad magnética, análisis geoquímicos de fluorescencia de rayos $X(X R F)$, mineralogía y contenido en carbono orgánico e inorgánico (TOC/TIC). El modelo cronológico se ha realizado combinando dataciones $A M S{ }^{14} \mathrm{C}$ en los $20 \mathrm{~m}$ superiores de sedimento y OSL (Luminiscencia Óptica Estimulada) en los $54 \mathrm{~m}$ restantes. La secuencia sedimentaria está esencialmente formada por la alternancia de fases con mayor desarrollo de humedales y turberas, con otras de depósitos distales de abanicos aluviales y episodios de lago carbonatado. Los resultados obtenidos hasta el momento confirman que el registro del Cañizar de Villarquemado responde a unas condiciones relativamente húmedas durante un probable MIS6-MIS5, con variaciones en la vegetación regional (esencialmente dominada por Juniperus) que aparecen siempre muy determinadas por la continentalidad de la zona, y que resultan atípicas para lo esperable durante un episodio interglacial como el Eemiense. De todos modos, los márgenes de error adscritos al modelo de edad impiden ciertas precisiones y aseveraciones al respecto. Durante el MIS3 se registra el periodo más árido de toda la secuencia, como confirman los resultados sedimentológicos y los polínicos de algunos intervalos ya disponibles. Sin embargo el MIS2 presenta una gran variabilidad tanto a nivel local, como indican las distintas asociaciones de facies y la 
vegetación acuática, como a nivel regional, como se deduce de la cubierta vegetal general. El comienzo del Holoceno se registra de manera muy abrupta en cuanto a cambios paleohidrológicos, pero mostrando de nuevo cierta resiliencia de la vegetación, dominada esta vez por pinos y no por Juniperus como en la base de la secuencia, y muy adaptada a las condiciones de extrema continentalidad. El posterior desarrollo de quercíneas junto con la presencia de pinos, apunta situaciones climáticas muy diferentes entre un posible MIS5 y el MIS1.

\section{Paleoenvironmental reconstruction of the last glacial cycle in the continental Iberia: the Cañizar de Villarquemado sequence (Teruel)}

ABSTRACT. El Cañizar de Villarquemado sequence (North-eastern Iberian Peninsula) stands as an exceptional palaeoenvironmental record for the last 130000 years. The preliminary multidisciplinary study carried out so far include pollen and sedimentological analyses, comprising geochemical, $X$ ray fluorescence $(X R F)$, mineralogical, organic and inorganic content (TOC/TIC) analyses. The chronological model has been established combining $A M S^{14} \mathrm{C}$ on the first $20 \mathrm{~m}$ of sediment and OSL methods on the remaining $54 \mathrm{~m}$ to the bottom. The sedimentary sequence is essentially formed by alternating facies of peatbogs and wetlands with those of distal alluvial fan deposits and others of carbonate lake. The results currently obtained support relatively humid conditions at El Cañizar de Villarquemado during a likely MIS6-MIS5 period, as errors linked to the age model prevent us to guarantee precisely the period boundaries. Despite indications of humidity, the regional vegetation variations during the MIS6-MIS5 period, essentially represented by Juniperus, were constrained by the intense continentality of the area, resulting in unexpected pollen assemblages for an interglacial period as the Eemian. The most arid phase of the sequence is registered during the MIS3, as shown by the available sedimentological and palynological results, while the MIS 2 presents greater local and regional variability as proved by the sedimentary facies and aquatics and the other pollen spectra, respectively. The Holocene presents an abrupt onset regarding palaeohydrology while terrestrial vegetation, dominated by pine instead than Juniperus as in the sequence's bottom, show a resilient response as these are well adapted communities to extreme continentality. The later development of both, evergreen and marcescent Quercus, together with the presence of pine woodland, indicate very different climatic conditions between the MIS5 and MIS1 interglacial periods.

Palabras clave: variabilidad hidrológica, continentalidad, resiliencia, aridez, Eemiense, Holoceno, último ciclo glacial.

Key words: hydrological variability, continentality, resilience, aridity, Eemian, Holocene, last glacial cycle.

Enviado el 25 de octubre de 2012

Aceptado el 20 de diciembre de 2012

*Correspondencia: Instituto Pirenaico de Ecología, CSIC, Avda. Montañana 1005, 50059 Zaragoza. E-mail: pgonzal@ipe.csic.es 


\section{Introducción}

El estudio paleoambiental del último ciclo glacial-interglacial resulta esencial para la comprensión del actual Cambio Global, tal y como se ha puesto de manifiesto en diferentes ámbitos científicos involucrados en el estudio del cambio climático (IPCC, 2007). Analizar periodos cronológicos largos permite el estudio de la dinámica de los ecosistemas en momentos muy diferentes, incluyendo circunstancias climáticas similares a las actuales, pero con particularidades distintas, como el hecho de contar o no contar con la impronta de la actividad humana como agente modulador del paisaje. Sin embargo, en el sur de Europa apenas existen registros continentales que proporcionen información paleoecológica para el intervalo temporal existente entre el Eemiense y el Holoceno (e.g., Ioannina: Tzedakis et al., 2003; Ohrid: Lézine et al., 2010; Vogel et al., 2010). Este fenómeno se acentúa en la Península Ibérica (PI), como documentan González-Sampériz et al. (2010) y Moreno et al. (2012). Teniendo en cuenta el notorio dinamismo del último ciclo glacial (74-11.6 ka cal BP) en términos de la variabilidad climática detectada a escala submilenaria -los llamados ciclos de Dansgaard/Oeschger (D/O), (Dansgaard et al., 1993)-, que presentan abruptos calentamientos y enfriamientos graduales (Wolff et al., 2010), resulta de suma importancia investigar la respuesta ecológica en las diferentes regiones de la Península, y dentro de un marco temporal continuo. En el actual escenario de Cambio Global (van Kolfschoten et al., 2003; Constanza et al., 2007), las áreas de la región Mediterránea son más vulnerables a la dinámica climática (Solomon et al., 2007) y por tanto, deben ser tomadas como sistema modelo dentro de la agenda geocientífica.

Durante las últimas décadas, la literatura pertinente viene proporcionando datos que completan ciertas lagunas de información, pero desgraciadamente, los mecanismos que generan la variabilidad climática, las teleconexiones que transfieren las señales climáticas por todo el planeta, y el impacto que los cambios climáticos rápidos tienen en los ecosistemas terrestres, aún están lejos de ser comprendidos en toda su magnitud (Broecker, 2000). De hecho, sabemos por ejemplo que algunos eventos de este último ciclo interglacial-glacial no han sido ni sincrónicos ni de la misma naturaleza en toda Europa, como es el caso del Eemiense en el interior continental (Sier et al., 2011), o del máximo avance de los glaciares en varias latitudes (Hughes y Woodward, 2008), incluyendo la PI (García-Ruiz et al., 2010). También aquí, los escasos datos paleoclimáticos disponibles sobre el Eemiense presentan ciertas discordancias en términos de, por ejemplo, el desarrollo de espeleotemas, ya que ciertas secuencias muestran un claro retraso cronológico y no registran su máximo crecimiento durante el MIS5e como sería esperable, sino que suceden en torno al MIS5c (Moreno et al., este volumen). Y es que a pesar de lo que se consideró hace unos años, el MIS5 y el Eemiense no son cronológicamente equivalentes. En particular, el MIS5 presenta mayor variabilidad de la que se había sospechado. Tras una notable controversia científica (Shackleton, 1969; Woillard y Mook, 1982; Guiot et al., 1989; Kukla et al., 1997; Chapman y Shackleton, 1999; Forsström, 2001; Tzedakis, 2003; Hodge et al., 2008), parece que ya existe cierto consenso sobre la duración del último interglacial, la cual sería aproximadamente de 16000 ó 17000 años, situándose entre 128000 y 112000 años BP. Esto implica una corres- 
pondencia en la mayoría de las secuencias con el segmento isotópico MIS5e-5d (Brauer et al., 2007), si bien hay alguna pequeña desviación local (Sier et al., 2011).

Al margen de su cronología, el Eemiense incluye otros aspectos merecedores de discusión. En particular, existen discrepancias sobre el alcance de las fluctuaciones en el nivel del mar (Waelbroeck et al., 2002; Dabrio et al., 2011), evolución de gradientes hídricos (Guan et al., 2007; Nikolova et al., 2012) y variaciones latitudinales del mosaico vegetal. Estas discrepancias mantienen viva la dialéctica sobre el nivel de similitud entre los dos últimos interglaciales, el Eemiense y el Holoceno (Ganolski y Robinson, 2011), particularmente relevante en cuanto a las posibles predicciones sobre el clima futuro. En términos de vegetación, por ejemplo, durante el Eemiense europeo es típica la expansión interglaciar de caducifolios (principalmente Corylus y Carpinus: de Beaulieu y Reille, 1992a,b; Ponsand et al., 1992; Muller, 2000; Drescher-Schneider, 2000; Muller et al., 2003; Satkunas et al., 2003; Tzedakis et al., 2003; Pini et al., 2009; Binka et al., 2011), así como un importante desarrollo paralelo del componente mediterráneo más hacia el sur (Quercus y Olea: Follieri et al., 1988; Pons y Reille, 1988; Carrión, 1992; Guiot y Cheddadi, 2004; Fernández et al., 2007). De hecho, en algunas secuencias, los valores que llega a alcanzar Olea en torno a 125000 BP por ejemplo, son incluso más altos que durante el Holoceno. La principal causa aludida para explicar el matiz termófilo que indica este taxón ha sido la coincidencia con el máximo de insolación interglacial (Tzedakis, 1994; Magri y Tzedakis, 2000; Klotz et al., 2003), que sin embargo implicaría también un incremento de la continentalidad y las temperaturas extremas (tanto invernales como estivales), con el consecuente aumento de la evapotranspiración (Braconnot et al., 2008), que no siempre se han tenido en cuenta ante la ausencia de datos del interior continental, donde este efecto sería particularmente acusado.

En este marco conceptual, la posibilidad de observar la dinámica de la vegetación y la evolución paleohidrológica de los últimos ca. 130000 años de historia, en una región sin datos al respecto hasta el momento como es el cuadrante nororiental de la PI, hacen de la secuencia presentada en este trabajo, El Cañizar de Villarquemado, un registro excepcional. Teniendo en cuenta que se localiza en un área Mediterránea con una importante influencia continental, partimos de la ausencia de los mencionados sesgos termófilos que afectan a las áreas más meridionales y costeras de las penínsulas del Mediterráneo occidental, aumentando por ello el interés de la secuencia, ya que los datos obtenidos en este registro resultan pioneros en cuanto a su extensión y resolución temporal. En este sentido, resulta de interés la cuestión de si el Eemiense presenta los mismos patrones climáticos que el Holoceno, y hasta qué punto se asemejan, porque ¿fue realmente más templado y húmedo el Eemiense que el presente interglacial?, ¿qué influencia tuvo el máximo de insolación del MIS5e en áreas de fuerte continentalidad como la de Villarquemado? Por otra parte, ¿cómo fue el advenimiento de la última glaciación, brusco o progresivo?, ¿qué características climáticas determinaron el MIS4?, ¿están relacionadas con el máximo avance de los glaciares en las montañas de la PI?, ¿qué periodo del último ciclo glacial fue el más árido?, ¿y el más húmedo? Aunque no estamos aún en disposición de responder satisfactoriamente a todas estas preguntas, en este trabajo presentamos resultados pre- 
liminares que pueden ayudarnos a ganar foco dentro de la problemática general y algo de luz sobre cuestiones particulares.

\section{Situación geográfica}

La laguna del Cañizar de Villaquemado (Teruel) está situada en el valle del Alto Jiloca, a $40^{\circ} 30^{\prime} \mathrm{N}$ y $1^{\circ} 17^{\prime} \mathrm{W}$. Dentro del ámbito geográfico-geológico de la Cordillera Ibérica, se localiza en la unidad geomorfológica conocida como "Fosa del Jiloca", a una altitud de 989 m s.n.m., entre las parameras de la Sierra de Albarracín al oeste y la crestería de Sierra de Palomera al este (Fig. 1).

La depresión del Jiloca tiene $60 \mathrm{~km}$ de largo y de 6 a $10 \mathrm{~km}$ de ancho. Se trata de una cuenca endorreica en su parte sur, determinada por fallas normales al este (Calamocha, Palomera y Concud) que crean una semi-fosa de actividad neotectónica Plio-Cuaternaria de carácter distensivo (Simón, 1983, AEQUA, 1993). Además, la conformación de la cuenca está asociada a un rebajamiento erosivo subkárstico de tipo polje, probablemente iniciado por la mencionada actividad neotectónica (Gracia et al., 2003; Gutiérrez et al., 2012; Gutiérrez et al., 2008; Lafuente et al.2010). Su fondo aparece modelado, casi en su totalidad, por una superficie de glacis desarrollada sobre los depósitos de piedemonte del Plioceno Superior y Cuaternario. Geológicamente, la depresión pertenece a una serie de cuencas intramontañosas desarrolladas en la Cordillera Ibérica durante el segundo episodio extensional que se inició en el Plioceno Superior (Simón, 1989; Simón et al., 2010; Casas-Sáinz y de Vicente, 2009). El cambio en las condiciones de endorreísmo a exorreísmo se produjo en estas depresiones durante el Neógeno y el Plio-Cuaternario (Gutiérrez y Gracia, 1997), cuando las cuencas fueron capturadas por la red de drenaje externa y se erosionaron las cabeceras. El río Jiloca capturó en primer lugar el semigraben de Daroca, y posteriormente la depresión del Jiloca, situada más al sur. Sin embargo, en el sector centro-sur de esta depresión se mantuvieron condiciones endorreicas en las que se desarrolló la Laguna del Cañizar, hasta que fue drenada artificialmente varias veces a lo largo de la historia, y definitivamente en el siglo XVIII (Rubio, 2004).

La laguna está situada sobre depósitos Cuaternarios lacustres afectados por aportes de grandes abanicos aluviales con materiales procedentes del Sistema Ibérico. Estos materiales son esencialmente mesozoicos (calizas y dolomías del Jurásico que forman el gran acuífero que alimenta los manantiales que nutren a la laguna), con algunos restos de afloramientos del Ordovícico y del Silúrico al suroeste y noroeste (formados por pizarras, areniscas, grauvacas y cuarcitas), y del Permo-Trías (con lutitas, areniscas, brechas y conglomerados, dolomías, margas y limolitas), circundando al Ordovícico y Silúrico y cubriendo las litologías Mesozoicas predominantes (Fig. 1D).

Como se ha mencionado anteriormente, el Cañizar de Villarquemado era un gran humedal de entre 10 y $11 \mathrm{~km}^{2}$, que fue drenado sistemáticamente durante siglos, consiguiendo su desecación completa en el siglo XVIII aduciendo razones de higiene y de salud pública, principalmente por las fiebres palúdicas (Rubio, 2004). En el momento de su desecación, la máxima zona inundada ocupaba $11.3 \mathrm{~km}^{2}$, y tenía una profundidad 

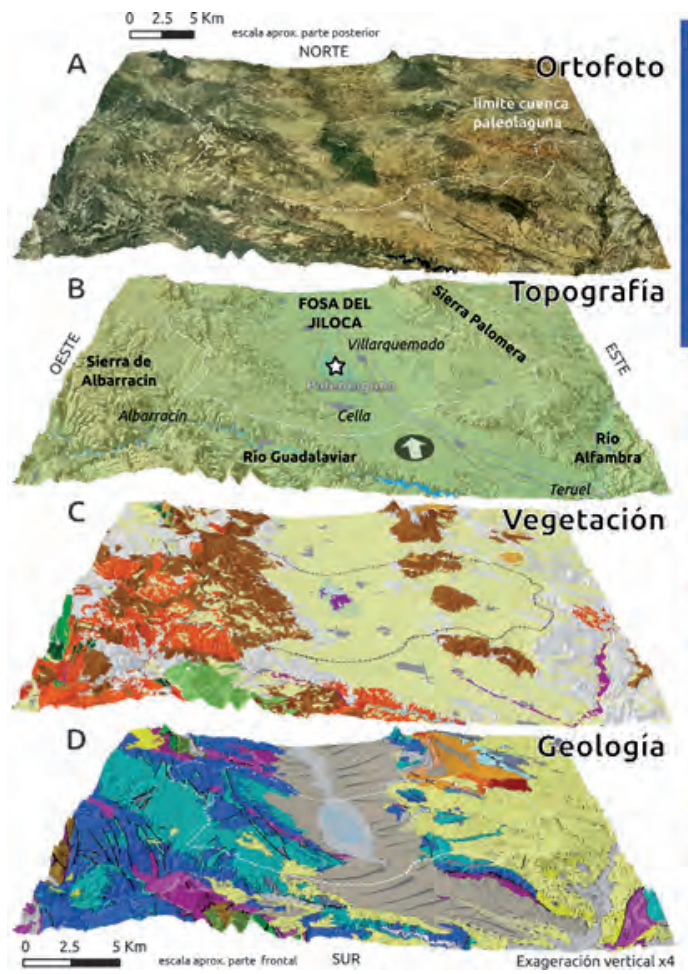

Leyenda

战 sondeo

- fronteras nacionales

-... limites CCAA

- rios

altitud (m.):

and 0

500

- 1000

1500
2000

2500

표 2500

$\square 3500$

Figura 1. Localización general de la laguna del Cañizar de Villarquemado: vista en 3D del área de estudio, ortofoto $(A)$, base topográfica $(B)$, principales formaciones vegetales $(C)$ y geología $(D)$.

media de entre $1.7 \mathrm{~m}$ y $2.8 \mathrm{~m}$ de lámina de agua, lo que le confería una capacidad de $18.8 \mathrm{hm}^{3}$ (Rubio, 2004). La desecación se realizó, principalmente, mediante la construcción de un canal de drenaje aún existente llamado "Acequia Madre”. Este canal tiene $45 \mathrm{~km}$ de largo y une Cella con el Alto de Singra, que es el límite de la cuenca endorreica del humedal. En la actualidad, se ha recuperado gran parte de la superficie que ocupaba antiguamente el humedal.

La cuenca del humedal del Cañizar presenta un clima Mediterráneo continental con una temperatura media anual de $10^{\circ} \mathrm{C}$ pero con una diferencia estacional de temperatura de $15^{\circ} \mathrm{C}$ y una precipitación media anual en torno a los $400 \mathrm{~mm}$, aumentando ligeramente en los márgenes del valle y disminuyendo hacia el extremo meridional (López Martín et al., 2007). Esta escasez de precipitaciones se explica por su localización interior y la presencia de áreas montañosas del Sistema Ibérico en su perímetro. Ambos hechos dificultan la llegada de masas de aire húmedo y frentes que aporten precipitación. Las perturbaciones de origen Atlántico que vienen desde el noroeste llegan muy debilitadas, y las sierras de Gúdar-Maestrazgo frenan las masas húmedas de procedencia mediterránea. Por su parte, los temporales del suroeste se ven obligados 
Serie de datos 1946-2002

\begin{tabular}{c|c|c|c} 
& $\mathrm{T}\left({ }^{\circ} \mathrm{C}\right)$ & $\mathrm{P}(\mathrm{mm})$ & ETF (mm) \\
\hline Ene & 2,70 & 18,35 & 9,40 \\
Feb & 4,09 & 16,49 & 17,10 \\
Mar & 6,83 & 22,74 & 41,18 \\
Abr & 9,11 & 34,26 & 60,74 \\
May & 13,42 & 56,12 & 90,56 \\
Jun & 18,00 & 45,68 & 118,41 \\
Jul & 21,60 & 24,49 & 142,40 \\
Ago & 20,93 & 32,45 & 122,99 \\
Sep & 16,96 & 36,34 & 62,41 \\
Oct & 11,56 & 38,14 & 32,73 \\
Nov & 6,36 & 27,74 & 11,70 \\
Dic & 3,36 & 24,20 & 7,15 \\
\hline Anual & $\mathbf{1 1 , 2}$ & 377,0 & 725,8
\end{tabular}

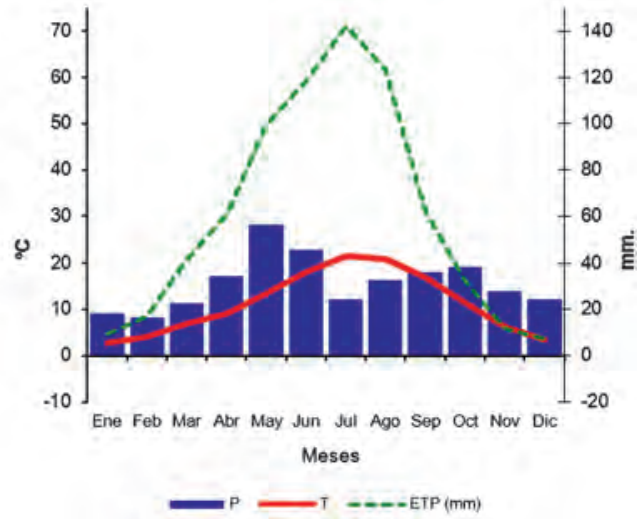

Figura 2. Climodiagrama de la estación meteorológica de Cella, ubicada en el entorno de la laguna del Cañizar de Villarquemado (Teruel). Fuente: Valores de temperaturas y precipitación procedentes del Atlas Climático de Aragón (López Martín et al., 2007) y cálculo de ETP según el método híbrido de Penman-Blandey y Criddle, con los valores $K$ de la estación de Teruel (Montero de Burgos et al., 1983). Los valores alcanzados por la evapotranspiración potencial (en verde), reflejan el importante déficit hídrico que registra la zona.

a atravesar casi toda la Península y dejan abundantes precipitaciones en el sector occidental del Sistema Ibérico, pero cuando alcanzan las tierras del Jiloca también han sufrido un fuerte proceso de desgaste reforzado por el conjunto montañoso de las Sierras de Albarracín, Montes Universales y Sierra Menera, que provoca un efecto de sombra pluviométrica (del Valle, 1993). Los registros climáticos de la estación de Cella (Fig. 2), ubicada junto a la laguna, evidencian el carácter continental del área, con temperaturas contrastadas, un régimen pluviométrico escaso de $377 \mathrm{~mm}$ anuales, con un máximo primaveral y otro secundario otoñal, y sobre todo, unos altos valores de evapotranspiración potencial en los meses centrales del año, lo que se traduce necesariamente en unos fuertes déficits hídricos en el lugar que determinan y limitan el desarrollo de la vegetación.

En cuanto a la vegetación del entorno (Fig. 1C), las zonas bajas de la cuenca en la que se localiza la laguna del Cañizar de Villarquemado, cuando no están ocupadas por cultivos, presentan carrascales (Quercus rotundifolia) y quejigares $(Q$. faginea), mientras que en las zonas altas más alejadas encontramos melojos ( $Q$. pyrenaica) y en las parameras calcáreas, sabinares (Juniperus phoenicea, J. thurifera) y pino laricio (Pinus nigra). Sobre areniscas se pueden observar formaciones de pino rodeno (Pinus pinaster) con jaras (Cistus spp.), que por encima de $1500 \mathrm{~m}$ dan paso a formaciones orófilas de Pinus sylvestris con Buxus sempervirens, Juniperus sabina, J. communis, Arctostaphylos uva-ursi, Erinacea anthyllis, Berberis vulgaris y Amelanchier ovalis, entre otros. Los pastos en los claros de los bosques son principalmente de Festuca gauthieri en 
zonas relativamente sombrías, y de Koeleria vallesiana y Festuca hystrix en las zonas más soleadas (Blanco Castro et al., 1997).

Como hecho destacable se puede señalar la presencia de Pinus uncinata en las cumbres de Gúdar, en torno a los 1900-2000 m, especie bien adaptada a la fuerte innivación y frío invernal. En zonas expuestas semiáridas y con suelos esqueléticos, encontramos una gran diversidad de plantas herbáceas y xerofíticas, entre las que predominan Thymus vulgaris, Rosmarinus officinalis, Lavandula latifolia, Satureja obovata, Genista scorpius, Ephedra fragilis, Artemisia herba-alba, Artemisia assoana, Santolina chamaecyparissus, Stipa tenacissima, Salsola kali y Salsola vermiculata. Por último, cabe señalar las formaciones riparias con Populus, Ulmus, Fraxinus y Salix, de mayor o menor extensión en función de la importancia de los valles. Además, como vegetación típica dominante de humedales, se desarrollan amplias formaciones de carrizos (Phragmites spp.) y aneas (Typha spp.). En suma, estamos ante un complejo mosaico vegetal con buena caracterización de pisos bioclimáticos, lo cual supone un valor añadido para la secuencia del Cañizar de Villarquemado como ecotono, particularmente sensible a la variación paleoambiental.

\section{Metodología}

En septiembre del año 2005 se realizó la campaña de recuperación de los 74 m de sondeo que conforman el registro sedimentario de la laguna de El Cañizar de Villarquemado, en Teruel. Se eligió un punto en la zona más deprimida de la cuenca lacustre, y dadas las características del sedimento y potencial del relleno, para la extracción del sondeo se utilizó un equipo de perforación de geotecnia mediante percusión. Todas las secciones del material obtenido en cada maniobra fueron convenientemente conservadas en papel de aluminio y tubos de PVC, y transportadas al laboratorio de sondeos del Instituto Pirenaico de Ecología (IPE-CSIC: http://www.ipe.csic.es), donde han sido analizadas y están conservadas en una cámara refrigerada a $4^{\circ} \mathrm{C}$.

El protocolo de actuación utilizado en la secuencia de Villarquemado es el habitual del equipo del IPE-CSIC, y sigue una metodología de análisis multi-indicador que comprende: i) la apertura longitudinal de los testigos; ii) el fotografiado en alta resolución de todas las secciones con un DMT® CoreScan; iii) la definición de las distintas facies sedimentarias a partir del análisis microscópico de frotis de sedimento (Schnurrenberger et al., 2003); iv) el estudio de las propiedades físicas (color, descripción y susceptibilidad magnética); v) el análisis multielemental con una resolución de $0.5 \mathrm{~cm}(\mathrm{~K}, \mathrm{Ca}, \mathrm{Ti}$, $\mathrm{Mn}, \mathrm{Fe}, \mathrm{Cr}, \mathrm{Sr}, \mathrm{Ba}, \mathrm{Cr}, \mathrm{Co}, \mathrm{Ni}, \mathrm{Zn}$ y Pb), mediante escáner de fluorescencia de rayos X (en este caso con el ITRAX core scanner del Large Lakes Observatory de la Universidad de Minnesota, USA); vi) la determinación del contenido en Carbono orgánico e inorgánico (TOC y TIC, respectivamente), cada $2 \mathrm{~cm}$, utilizando el LECO SC144DR del laboratorio del IPE-CSIC; vii) el análisis mineralógico en muestras discretas con un difractómetro de rayos X Philips PW1820 para conocer la abundancia relativa de determinados minerales y ayudar en la caracterización de facies sedimentarias; y viii) el análisis palinológico de muestras cada $10-20 \mathrm{~cm}$ en toda la secuencia, aumentando la 
resolución en determinados intervalos. Aquí cabe señalar que parte de la secuencia aún está en proceso de estudio y en este trabajo sólo se presentan resultados preliminares del techo y la base del registro. El protocolo seguido para el análisis del contenido en granos de polen y esporas ha sido el clásico establecido por Moore et al. (1991), sin incluir acetolisis, realizando una concentración a partir de líquido denso (Thoulet 2.0) para enriquecer las muestras en palinomorfos, y añadiendo dos pastillas de Lycopodium clavatum (concentración conocida) en cada muestra para testar el procesado físico-químico y posibilitar el cálculo de las concentraciones polínicas.

El modelo cronológico está basado en 12 dataciones radiocarbónicas a lo largo de los $20 \mathrm{~m}$ superiores del sondeo, lo que se corresponde con aproximadamente los últimos 40000 años de historia, mientras que en el resto de la secuencia se han distribuido 5 dataciones de Luminiscencia Ópticamente Estimulada (OSL) realizadas en la Universidad Autónoma de Madrid (Tabla 1). Los amplios márgenes de error asociados a las dataciones OSL hacen previsible que la base de la secuencia recoja un periodo impreciso entre el final del MIS6 y el MIS5 (130-110 ka BP). Actualmente están en proceso 10 nuevas dataciones OSL siguiendo la técnica single grain, que ayudarán a matizar la cronología. Las dataciones AMS C ${ }^{14}$ han sido calibradas con Calib 6.11 (Stuiver y Reimer, 1993) utilizando la curva de IntCal09 (Reimer et al., 2009) y el modelo de edad se ha elaborado en base a Heegard et al., (2005) (Fig. 3).

Tabla 1. Dataciones obtenidas hasta el momento en la secuencia del Cañizar de Villarquemado.

A lo largo de la potencia sedimentaria se han combinado la técnica de AMS realizada en el Poznán Radiocarbon Laboratory de Polonia, con dataciones de Luminiscencia Ópticamente Estimulada (OSL) realizadas en el Laboratorio de Datación y Radioquímica de la Universidad Autónoma de Madrid.

\begin{tabular}{|c|c|c|c|c|c|c|c|c|}
\hline $\mathrm{N}^{0}$ & $\begin{array}{l}\text { Código } \\
\text { laboratorio }\end{array}$ & Sigla & $\begin{array}{l}\text { Profundidad } \\
\text { (cm) }\end{array}$ & Material & Técnica & Edad & Error & $\begin{array}{c}\text { Fechas } \\
\text { calibradas }\end{array}$ \\
\hline 1 & Poz-18451 & VIL05-1B-1T-2,37 cm & 970 & sedimento orgánico & AMS & 3750 & 40 & $4.111+100$ \\
\hline 2 & Poz-18509 & VIL05-1B-1T-3, $39 \mathrm{~cm}$ & 1736 & sedimento orgánico & AMS & 7460 & 50 & $8.279+94$ \\
\hline 3 & Poz-18453 & VIL05-1B-1T-4, 41-42 & 2310 & sedimento orgánico & AMS & 9820 & 50 & $11.232+75$ \\
\hline 4 & Poz-15943 & VIL05-1B-1T-5, $54 \mathrm{~cm}$ & 3060 & sedimento orgánico & AMS & 11620 & 60 & $13.461+174$ \\
\hline 5 & Poz-18511 & VIL05-1B-1T-8, 32-35 cm & 4533 & sedimento orgánico & AMS & 15390 & 100 & $18.640+192$ \\
\hline 6 & Poz-15944 & VIL05-1B-1T-10, $15 \mathrm{~cm}$ & 5504 & sedimento orgánico & AMS & 18280 & 110 & $21.830+372$ \\
\hline 7 & Poz-15945 & VIL05-1B-1T-14, $1 \mathrm{~cm}$ & 7310 & sedimento orgánico & AMS & 21020 & 140 & $25.077+502$ \\
\hline 8 & Poz-15946 & VIL05-1B-1T-16, $5 \mathrm{~cm}$ & 8610 & sedimento orgánico & AMS & 22780 & 160 & $27.511+569$ \\
\hline 9 & Poz-23714 & VIL05-1B-1T-21, $13 \mathrm{~cm}$ & 11413 & sedimento orgánico & AMS & 25520 & 380 & $30.325+712$ \\
\hline 10 & Poz-15948 & VIL05-1B-1T-24, $12 \mathrm{~cm}$ & 13179 & sedimento orgánico & AMS & 27900 & 300 & $32.100+754$ \\
\hline 11 & Poz-17394 & VIL05-1B-1T-28, $7 \mathrm{~cm}$ & 14935 & sedimento orgánico & AMS & 33300 & 800 & $38.009+1892$ \\
\hline 12 & MAD-5172SDA & VIL05-1B-1T-34 & 18321 & sedimento siliciclástico & OSL & 40421 & 3468 & \\
\hline 13 & Poz-17287 & VIL05-1B-1T- $35,44 \mathrm{~cm}$ & 19154 & sedimento orgánico & AMS & 36800 & 800 & $41.687+1348$ \\
\hline 14 & MAD-5173SDA & VIL05-1B-1T-92 & 48816 & sedimento siliciclástico & OSL & 72457 & 5152 & \\
\hline 15 & MAD-5196SDA & VIL05-1B-1T-103 & 53574 & sedimento siliciclástico & OSL & 93551 & 6816 & \\
\hline 16 & MAD-5200SDA & VIL05-1B-1T-145 & 71072 & sedimento siliciclástico & OSL & 115890 & 8996 & \\
\hline 17 & MAD-5203SDA & VIL05-1B-1T-149 & 73295 & sedimento siliciclástico & OSL & 120808 & 8209 & \\
\hline
\end{tabular}




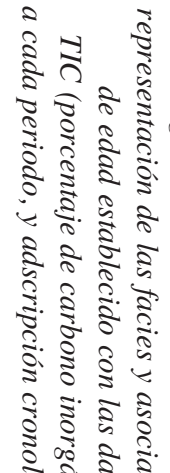

ㅇำ

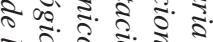

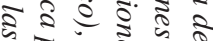
$5.75=\frac{1}{5}$ ป ڤ

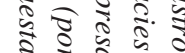
ㄷำ ร. ॠ

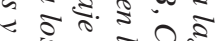

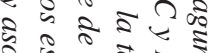

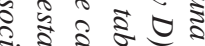
ร.

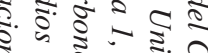
ฐ ร.5. द. ร. ร. 2 ริ

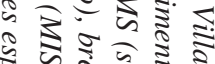

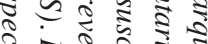
ร.

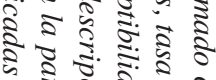

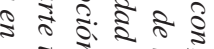

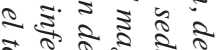

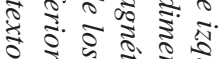

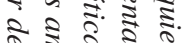

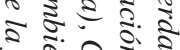

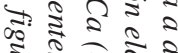

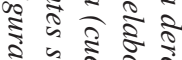
क ई ร ฐ. ฐ

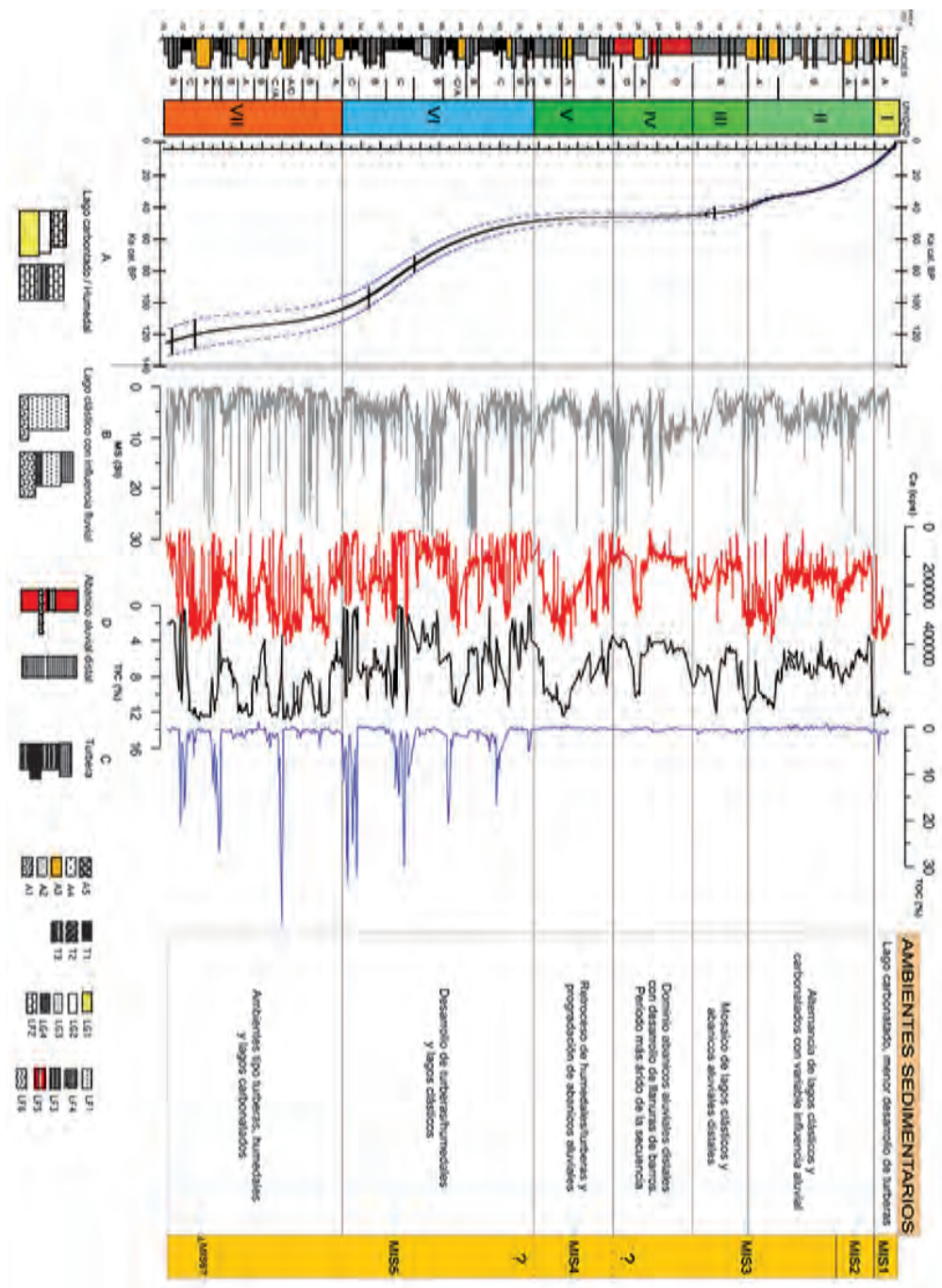
इ

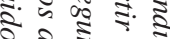
इ ริ ई ई

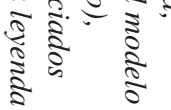




\section{Resultados}

El análisis sedimentológico incluye la composición geoquímica y mineralógica de los sedimentos y las observaciones al microscopio de frotis de sedimentos. Se han definido hasta 20 facies sedimentarias distribuidas en cuatro asociaciones (A, B, C y D: Fig. 3). A su vez, las facies se han agrupado en cuatro tipos en función de sus características granulométricas y composicionales. Las facies de arenas incluyen arenas grises (A1) y marrones (A2), de composición más siliciclástica, arenas amarillentas carbonatadas (A3), arenas con abundantes restos de turba (A4) y bioclásticas (A5). Las facies de limos gruesos son más siliciclásticas (LG4) o más finas y carbonatadas (LG1), con moteado rojizo (LG2) o gris (LG3). Las facies de limos finos son las más abundantes e incluyen limos grises (LF1), en ocasiones con moteados negros (LF4) o versicolores (LF6), limos color crema carbonatados (LF2), limos negros (LF3), limos rojos (LF5) y limos laminados grises-verdosos (LF7). Las facies de turba incluyen capas homogéneas masivas (T1), capas de limos turbosos (T2), agregados gruesos de turba (T3) y suelo actual (T4).

Las asociaciones de facies definidas son las siguientes:

- Asociación A "Lago carbonatado". Incluye facies de arenas (A2, A3, A5), limos gruesos (LG1) y finos (LF1, LF2, LF6), ocasionalmente con facies de limos turbosos (T2) y orgánicos (LF3), propias de ambientes de depósito con elevada producción de carbonatos (tipo pradera de caráceas y plataforma litoral) y ambientes mixtos carbonatados tipo lago carbonatado, humedal y abanico aluvial distal.

- Asociación B "Lago clástico". Incluye facies de arenas (A1), limos gruesos (LG2, LG3, LG4) y limos finos (LF1, LF4, LF6, LF7), de composición más siliciclástica y con características de ambientes litorales y de ambientes más distales, relativamente profundos.

- Asociación C “Turbera-humedal”. Incluye las facies de turba masiva, homogénea (T1) y depósitos de humedales orgánicos con desarrollo de suelos (T4), facies de retrabajamiento y redepósito de las capas de turba (A4, T2, T3), arenas bioclásticas (A5) y limos finos orgánicos (LF3).

- Asociación D “Abanico aluvial distal/ llanura de barros”. Incluye las facies de limos finos rojos (LF5), alternando con limos grises (LF4) y arenas grises (A1).

De acuerdo con la distribución de facies y de asociaciones de facies, se han individualizado siete unidades sedimentarias en el sondeo de la Laguna del Cañizar (Fig. 3): Unidad VII (74-56 m), caracterizada por ambiente de depósito de turberas, humedales y lagos carbonatados; Unidad VI (56-37 m), con asociaciones de facies de turbera/humedal y lagos clásticos; Unidad V (37-29 m), caracterizada por el retroceso de los humedales/turberas y la progradación de los abanicos aluviales sobre la cuenca de la laguna; Unidad IV (29-21 m), con dominio de los ambientes de abanicos aluviales distales y con el desarrollo de llanuras de barros; Unidad III (21-15 m), con facies de lagos clásticos y abanicos aluviales distales; Unidad II (15-3 m), formada por una alternancia de lagos clásticos y carbonatados con variable influencia aluvial; Unidad I (3-0 m), 
que representa un lago carbonatado con menor desarrollo de ambientes de turbera y humedales asociados.

Las dataciones OSL del intervalo comprendido entre 74-18 m establecen una cronología situada entre 130 41 ka BP (Tabla 1), pero los márgenes de error de cada datación impiden concretar si los metros inferiores corresponden al MIS6 o al MIS5, y definir los límites entre MIS5-MIS4 y MIS4-MIS3, por lo que, siendo conscientes de que disponemos tan sólo de resultados parciales de parte de los indicadores analizados, la cronología representada en la Fig. 3 y que se sigue a lo largo de la discusión de este trabajo, ha sido matizada en base a los límites de las unidades sedimentarias diferenciadas, tal como se señaló en Moreno et al. (2012). De este modo, las Unidades VII y VI quedarían incluidas dentro del MIS5, aunque teniendo en cuenta que, según el modelo de edad y sus márgenes de error, parte de la Unidad VII podría pertenecer al final del MIS6. Por añadidura, la Unidad V se asimilaría al MIS4, las Unidades IV, III y parte de la II se incluirían en el MIS3 y la parte superior de la Unidad II en el MIS2. Finalmente, la Unidad I correspondería al Holoceno (MIS1).

En cuanto a los datos palinológicos presentados en este trabajo, sólo incluyen una selección de taxa con el fin de observar las principales tendencias registradas en las muestras analizadas hasta el momento. En total, se han seleccionado siete curvas o grupos, a partir de los elementos dominantes actualmente en la vegetación regional y en la evolución local del humedal: i) Pinus total, incluyendo los espectros polínicos de pinos tanto de montaña-Eurosiberianos (tipo Pinus sylvestris-nigra) como los más Mediterráneos (tipo Pinus halepensis-pinaster); ii) Juniperus, ya que conforma importantes extensiones hoy en día (Fig. 1C) y ha sido protagonista indiscutible de los espectros en diversos periodos de la secuencia; iii) Quercus total, incluyendo los tipos polínicos perennes, caducos y marcescentes, ya que al tratarse de resultados preliminares no vamos a entrar en variaciones de temperatura y/o humedad de detalle en los diferentes periodos con resultados polínicos; iv) polen arbóreo total (AP) para dar una idea general de las variaciones de las masas forestales a escala regional; v) Poaceae, ya que son las herbáceas dominantes; vi) Artemisia y Chenopodiaceae, como principales indicadores del componente estépico del paisaje; vii) e Higro-hidrófitas totales $(\mathrm{HH})$, para una primera aproximación de la evolución paleohidrológica del humedal y su posible correspondencia con las facies sedimentarias identificadas. Los colores elegidos para la representación gráfica de estos taxa en el diagrama polínico (Fig. 4) se corresponden con los utilizados en el mapa de vegetación actual presentado en la Fig. $1 \mathrm{C}$, con idea de facilitar las comparaciones. Las curvas pertenecen exclusivamente al intervalo basal comprendido entre 73 y $56 \mathrm{~m}$, con una resolución media de análisis de $25 \mathrm{~cm}$, y a los $30 \mathrm{~m}$ superiores, con una resolución media de $20 \mathrm{~cm}$ (excepto en los $3 \mathrm{~m}$ superiores, correspondientes al Holoceno, donde la resolución aumenta a $2 \mathrm{~cm}$ ). La finalidad de esta selección de taxones e intervalos ha sido realizar entre ambos una comparativa general de ambientes y periodos climáticos distintos (posible interglacialglacial-interglacial). Quedan pendientes de analizar casi por completo los intervalos correspondientes a las Unidades sedimentarias VI y V. 


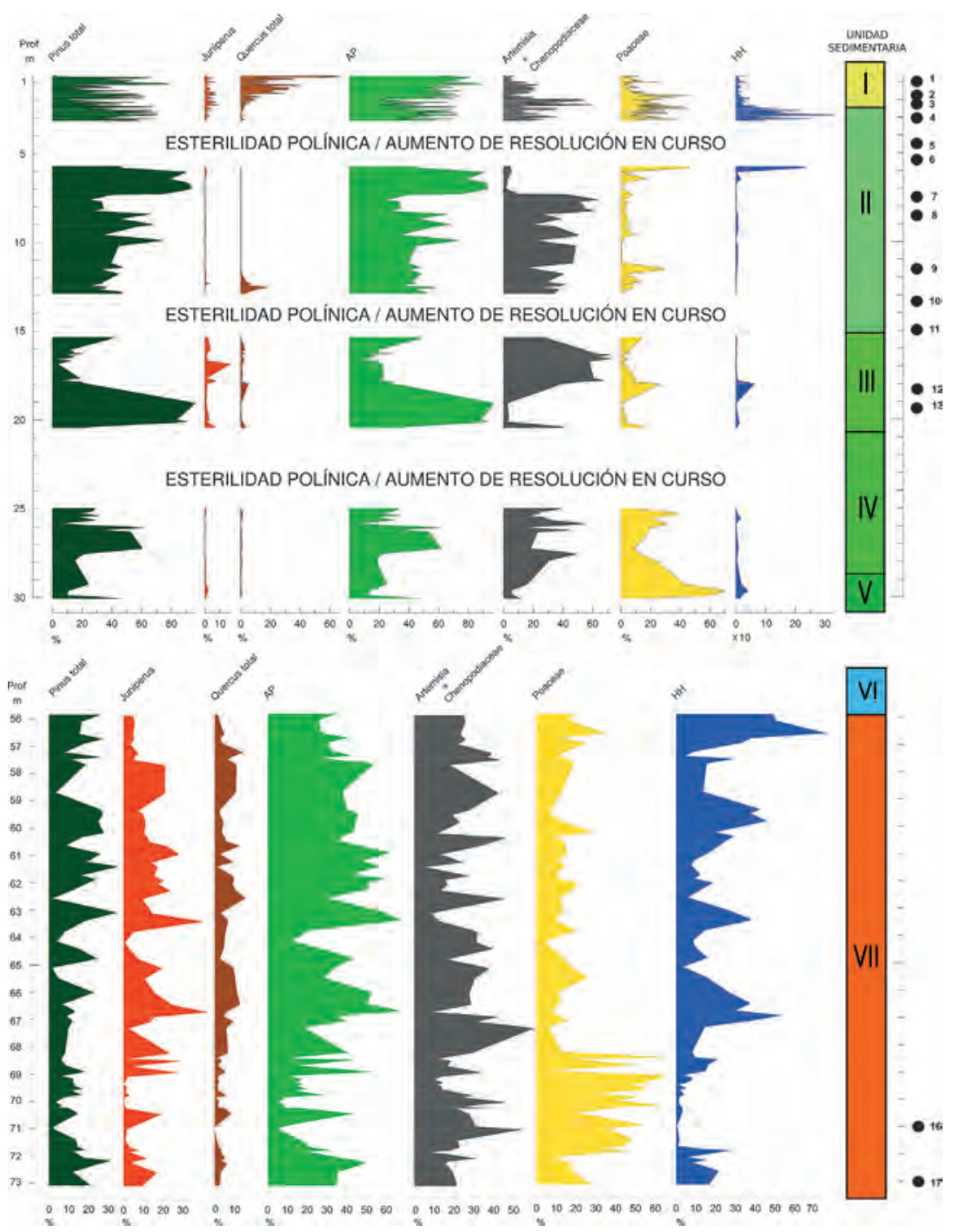

Figura 4. Diagrama polínico con los intervalos analizados hasta el momento en el techo y la base de la secuencia del Cañizar de Villarquemado. Se han representado una selección de taxones o grupos, con el fin de comparar las distintas Unidades Sedimentarias del registro.

Los colores adoptados para las distintas curvas son los mismos que se han utilizado en el mapa de vegetación de la Figura 1 para las especies dominantes en la región en la actualidad. La curva de AP está compuesta por Pinus, Juniperus, Abies, Cedrus, Picea, Taxus, Betula, Corylus, Alnus, Carpinus, Salix, Populus, Acer, Fraxinus, Fagus, Tilia, Juglans, Castanea, Olea, Quercus, Pterocarya. El grupo de HH está formado por Ranunculus, Thalictrum, Lythrum, Juncus, Utricularia, Cyperaceae, Typhaceae, Pedicularis, Sparganium, Lemna, Nymphaea, Myriophyllum, Potamogeton, Isoetes, Alisma. Las dataciones obtenidas hasta el momento están marcadas estratigráficamente en la figura con un punto negro a la derecha de las Unidades Sedimentarias, y responden a la numeración establecida en la Tabla 1. 


\section{Discusión}

5.1. ¿Un Eemiense atípico, su preludio o el comienzo del último ciclo glacial? MIS6, MIS5 y MIS4 en la secuencia de Villarquemado

La secuencia sedimentológica del Cañizar de Villarquemado se compone, básicamente, de facies de turbera, abanicos aluviales y depósitos de lago carbonatado, que durante la evolución de la cuenca a lo largo de los últimos ca. 130000 años de historia se han ido sucediendo en un mosaico variable de estos tres ambientes sedimentarios, controlados por el clima, la subsidencia y la dinámica con los abanicos aluviales originados en las sierras próximas (Fig. 1D y Fig. 3). En este sentido, el desarrollo de un lago carbonatado (con altos contenidos de Ca y TIC y valores bajos de MS), representa niveles de lago más altos que los característicos de turbera (valores altos en TOC, bajos en MS), mientras que los depósitos de abanicos aluviales (bajo contenido de carbonatos y TOC, alta MS), representan periodos con los niveles hidrológicos más bajos. Así, en la secuencia de Villarquemado (Fig. 3), los valores de TOC son más altos durante el Holoceno (Unidad I, 3-0 m) y MIS5 (Unidades VII y VI, 74-37 m) con el desarrollo más significativo de los humedales de toda la secuencia. Durante la Unidad VII (74-56 m), se registran numerosas facies de turba con frecuentes intercalaciones de facies carbonatadas lacustres, indicando una fase de expansión de la laguna y de los humedales asociados, mientras que en la Unidad VI (56-37 m), se observa un mayor desarrollo de depósitos de turba intercalados con sedimentos lacustres y aluviales clásticos, sugiriendo un mayor desarrollo de lagos clásticos y humedales. Un cambio deposicional significativo se registra al inicio de la Unidad V (29-37 m), con la reducción de la extensión de los humedales y la progradación de los abanicos aluviales distales indicativos de una tendencia hacia niveles hidrológicos más bajos (Moreno et al., 2012). Según nuestro actual modelo de edad, esta última unidad se correspondería con el MIS4.

Palinológicamente, de los espectros obtenidos hasta el momento podemos obtener información tanto de carácter regional como local, basada en los contenidos de Higrohidrófitas-HH. En este último grupo (Fig. 4), se observan importantes fluctuaciones más o menos acordes con el desarrollo de una turbera alternante con niveles de lago carbonatado. Encontramos intervalos sin apenas representación de $\mathrm{HH}$, especialmente entre los 72 y 69 m de profundidad, cuando se registran las mayores proporciones de Poaceae. Teniendo en cuenta la toponimia y aspecto actual de la laguna del Cañizar de Villarquemado, Phragmites ha podido jugar un papel esencial en la composición de la curva de Poaceae a lo largo de toda la secuencia, pero palinológicamente no podemos diferenciarlo del resto de géneros que conforman esta amplia familia, lo que dificulta la interpretación del registro vegetal. En cualquier caso, su evolución en antifase a lo largo de la Unidad VII, podría estar reflejando la sustitución del carrizal por otro tipo de comunidades acuáticas, en línea con la alternancia de las distintas facies sedimentarias.

Es en lo referente a la vegetación terrestre cuando nos encontramos una composición florística y unas proporciones de taxa dominantes, bastante alejadas de lo esperado para un periodo interglacial como el MIS5, en comparación tanto con los escasos datos existentes en la cuenca Mediterránea y en la PI, como con los espectros de los que se disponen para el Holoceno en esta misma secuencia. La base de Villarquemado (73-56 m de 
profundidad, Unidad VII), muestra unas proporciones de polen arbóreo (AP) relativamente bajas (30\% de media con máximos puntuales del 60\% a 66.5 y $63 \mathrm{~m}$ y mínimos del $10 \%$ a $71 \mathrm{~m}$, por ejemplo). Esta cubierta arbóreo-arbustiva está dominada por coníferas, destacando los valores de Juniperus, que llegan a alcanzar el $40 \%$ en los máximos de AP. La presencia de Quercus puede considerarse escasa (apenas supera el 10\% en los intervalos de máxima representación), máxime en comparación con los valores que se llegan a alcanzar durante el Holoceno (más del 50\% en el techo de la secuencia). Las herbáceas de carácter estépico tipo Artemisia y Chenopodiaceae llegan a un 20-30\% de media, aunque también presentan fuertes fluctuaciones. Obviamente, esta composición vegetal y sus proporciones no se corresponden con los patrones conocidos hasta el momento en las secuencias palinológicas de referencia europeas para el Eemiense ó MIS5e (Follieri et al., 1988; Pons y Reille, 1988; Sánchez-Goñi et al., 1999; Tzedakis et al., 2003; Muller et al., 2003) que, no obstante, no se localizan en áreas climáticas mediterráneas con una influencia continental tan fuerte como nuestra área de estudio.

En este sentido, y teniendo en cuenta el margen de error de las dataciones OSL de las que disponemos para nuestro modelo de edad, que no permiten precisar si la base de la secuencia se adscribe cronológicamente al final del MIS6 ó al MIS5, cabe mencionar la similitud existente entre los espectros polínicos obtenidos en la base del registro de Villarquemado y los de la LPAZ22 de la secuencia de Lago Grande di Monticchio (Allen y Huntley, 2009), datada en 133-127 ka BP, es decir, al final de la penúltima glaciación y transición al interglacial Eemiense (Brauer et al., 2007). En Monticchio, Juniperus alcanza valores en torno al 20\% de media, Pinus alrededor del 10\%, Quercus entre $5-10 \%$, Poaceae fluctúa entre 20 y $40 \%$, y Artemisia y Chenopodiaceae suman proporciones de 30-40\% (Fig. 5), valores todos ellos muy semejantes a los de la Unidad VII de Villarquemado. Las condiciones climáticas reconstruidas en Monticchio a partir de los mencionados espectros polínicos nos hablan de inviernos fríos y un pronunciado déficit hídrico estacional (Allen et al., 2002), asociado a veranos relativamente cálidos, como consecuencia de la insolación de verano, que aunque aún no llega a los máximos del Eemiense, sí es ligeramente superior a la actual (Laskar et al., 2004, Fig. 5). Los contrastes estacionales, y por lo tanto la continentalidad, serían ya muy notables durante el final del MIS6. La continentalidad que caracteriza al área de la laguna del Cañizar de Villarquemado (Fig. 2), justificaría un dominio de Juniperus en el estrato arbóreo-arbustivo, ya que este género está adaptado a extremos térmicos y a severas sequías estivales, especialmente en el caso de Juniperus thurifera, que es una especie de amplia distribución actualmente en la zona (Fig. 1). A tenor de los resultados obtenidos hasta el momento, esta situación no se repite en ningún otro periodo de la secuencia. Además de por el registro polínico, esta adscripción al final del MIS6 de la base de la secuencia resulta acorde con las facies sedimentarias anteriormente descritas, que apuntaban condiciones húmedas durante un periodo que parece estar caracterizado en el noreste de la PI por frío y alta disponibilidad hídrica, tal como plantean Benito et al. (2010) a partir de las fases de agradación de las terrazas fluviales del río Gállego.

No obstante, como se viene comentando a lo largo de este trabajo, al no poseer apenas datos comparativos para el MIS5 en el interior continental, no podemos desestimar del todo que nuestra Unidad VII responda a un Eemiense de carácter atípico en términos 


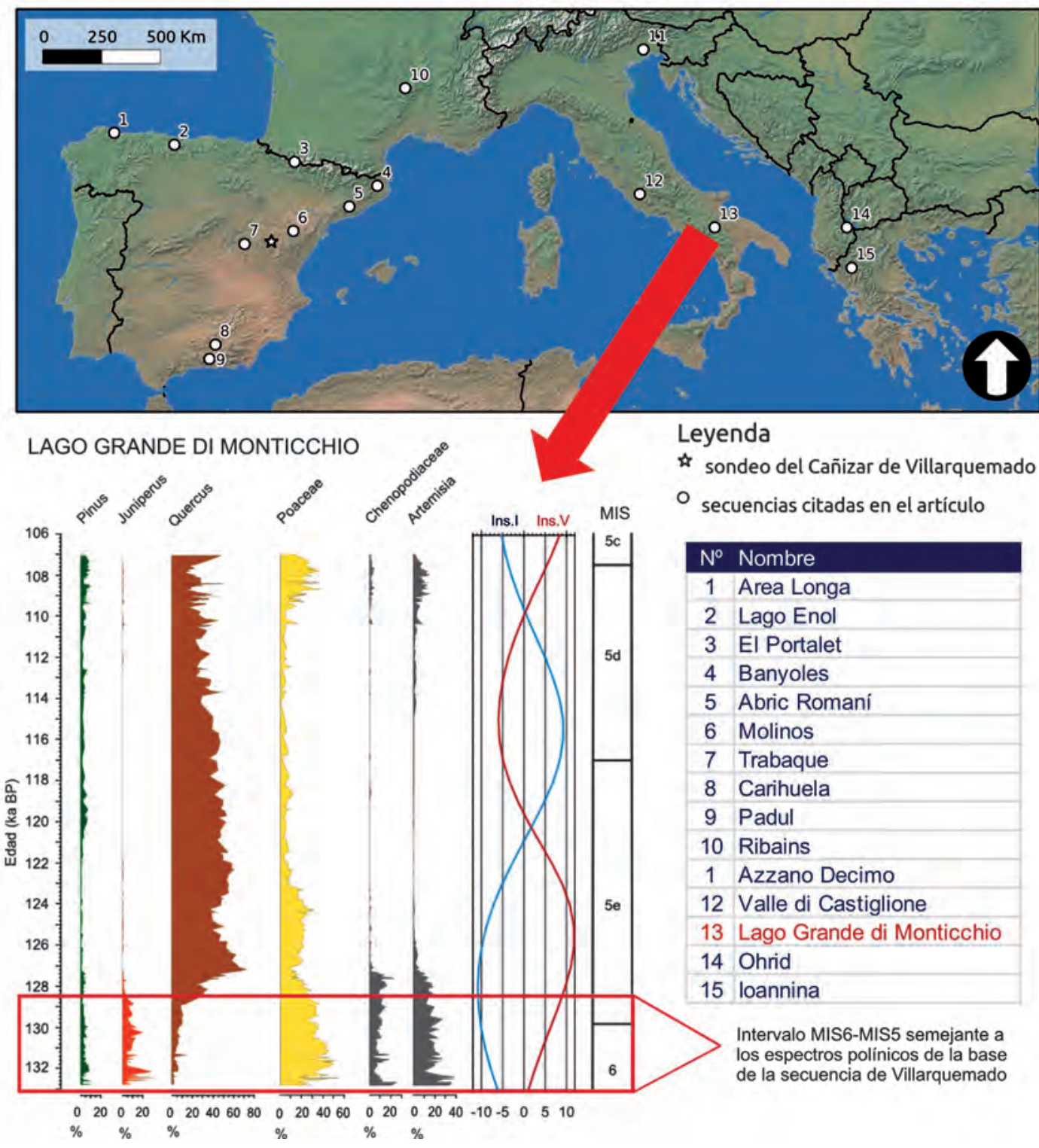

Figura 5. Mapa de la cuenca Mediterránea con la localización de las principales secuencias mencionadas en la discusión de este trabajo, y diagrama palinológico sintético (a partir de Allen y Huntley, 2009 modificado) de la base de la secuencia de Lago Grande di Monticchio (transición MIS6-MIS5), para observar la semejanza de los espectros polínicos (marcados con rectángulo rojo) con los obtenidos en la base de la secuencia del Cañizar de Villarquemado y que aparecen representados en la Figura 4. Las curvas de la insolación de invierno (Ins.I: línea azul) y verano (Ins.V: línea roja), representadas a la derecha del diagrama palinológico de Lago Grande di Monticchio, corresponden a valores calculados por Laskar et al. (2004) y son las incluidas en Allen y Huntley (2009). 
de expansión forestal. Los máximos de insolación de verano y mínimos de invierno reconstruidos para el MIS5e (Laskar et al., 2004), podrían haber determinado en una zona como el interior de Teruel, una formación vegetal resiliente y muy adaptada a extremos de aridez y temperatura, como los sabinares que crecen en la actualidad en las parameras de la región y del interior de la PI (Blanco-Castro et al., 1997). De este modo, y a pesar de las supuestas condiciones de mayor temperatura y humedad asociadas al anterior interglacial, el patrón de la vegetación de áreas muy continentales como nuestra zona de estudio, no respondería ni al dominio de caducifolios registrado en secuencias europeas, donde la evapotranspiración no es un factor limitante para la vegetación (Fig. 2), ni a las expansiones de Olea asociadas a las áreas costeras termófilas del sur de las penínsulas Mediterráneas. Además, al margen de que en la región se han establecido condiciones de máximos de humedad y elevadas temperaturas para el MIS5e en los depósitos tobáceos del Trabaque (Domínguez et al., 2011), nuevos datos en registros espeleotémicos de la Gruta del Cristal de Molinos (Moreno et al., este volumen), no apuntan el MIS5e como el periodo de máximo crecimiento de estalagmitas, sino el MIS5c, en línea con otros registros europeos continentales en los que la expansión forestal no alcanzaría su máximo desarrollo durante el MIS5e, sino con un retraso de, al menos, 5000 años (Sier et al., 2011), configurando así una compleja variabilidad climática dentro del MIS5.

Al carecer de resultados palinológicos para las Unidades VI y V, no estamos en disposición de confirmar ninguna de las dos hipótesis (MIS6 ó MIS5) para la adscripción cronológica de la Unidad VII de Villarquemado, sin embargo, resulta obvio que los espectros polínicos del techo de la secuencia, sin ninguna duda pertenecientes al Holoceno, no se asemejan en absoluto a los de la base, en el caso de corresponder ambos a periodos interglaciales.

Los únicos datos de polen que tenemos en la Unidad V corresponden a la transición a la Unidad IV. En estos niveles, se registran muy bajas proporciones de Artemisia y Chenopodiaceae (10\%) en comparación con los espectros anteriores y superiores (entre el 20 y el 50\%), un pico de Poaceae que supera el 60\%, semejante a los de la base de la secuencia (entre 72 y $69 \mathrm{~m}$ ) y una escasa proporción de $\mathrm{HH}$ (entre 2 y 5\%), que se mantendrá muy similar a lo largo de la Unidad IV, junto a una presencia arbórea fluctuante y dominada por el pino, con una ausencia casi total de Juniperus y Quercus, así como la recuperación del componente estépico. El resto de la Unidad IV (entre 25 y $21 \mathrm{~m}$ de profundidad), está sin analizar hasta el momento, pero los datos preliminares ya apuntan a unas condiciones claras de frío y aridez, con una muy escasa cubierta vegetal, en unos niveles en los que las facies sedimentarias indican que se trataría del periodo más árido de todo el registro (entre 29 y 21 m de profundidad), a pesar de que con nuestro actual modelo de edad, no podamos precisar si estas condiciones pertenecen al MIS4 o al comienzo del MIS3.

De los escasos datos existentes en la PI con cronologías semejantes, y procedentes de secuencias largas, debemos mencionar la secuencia litoral de Area Longa (Fig. 5), que abarca todo el intervalo de MIS5c-MIS4 (Gómez-Orellana et al., 2007). Obviamente, no se trata de espectros semejantes a los de Villarquemado, porque la localización geográfica de una secuencia y otra implican importantes diferencias en términos bioclimáticos y de composición vegetal. Sin embargo los patrones de cambio encontrados en 
este estudio indican condiciones ambientales comparables a las del Cañizar de Villarquemado. La base de este registro polínico está dominada por bosques caducifolios (Alnus, Quercus caducifolios, Corylus, Betula y Carpinus), incluyendo altas proporciones de Fagus. Durante el MIS4, altos porcentajes de Erica, Calluna y Poaceae indican en Area Longa la existencia de brezales y praderas con una baja abundancia de coníferas y persistencia de mesotermófilas como Quercus caducifolios, Corylus, Fagus, Carpinus, Ulmus e Ilex. La interpretación que hacen los autores es que, aunque el noroeste de la PI se vería afectado por el enfriamiento que estaba ocurriendo a nivel mundial durante el MIS4, el clima regional siguió siendo relativamente húmedo, basándose principalmente en los altos porcentajes de Ericaceae y Poaceae, y las bajas proporciones de taxa estépicos registradas (Artemisia, Chenopodiaceae). En Abric Romaní, en el noreste de la PI, los porcentajes de polen arbóreo de los depósitos más antiguos (atribuidos al MIS5a) llegan a un 40-60\%, y están dominados por pinos, pero con una continua presencia de Juniperus, Rhamnus, Quercus, Olea-Phillyrea, Betula, Fagus, Pistacia y otros taxones mesotermófilos. La transición al MIS4 representa una fase fría y húmeda, con taxones menos termófilos (Burjachs y Julià, 1994; Burjachs et al., 2012).

Por otra parte, los registros de glaciares del Pirineo Central (García-Ruíz et al., 2003, 2012; Pallàs et al., 2006; Lewis et al., 2009) apoyan la prevalencia de condiciones relativamente húmedas en la transición entre MIS5 y MIS4 en el norte de la PI. Las morrenas más externas del Pirineo Central español se han datado mediante OSL en $85 \pm 5$ ka (Lewis et al., 2009; Peña et al., 2004), situando el momento del "máximo avance glaciar” cerca de la transición entre MIS5 y MIS4 (García-Ruiz et al., 2010). Este escenario de bajas temperaturas y humedad significativa a lo largo del norte de la PI, y una disminución gradual de la humedad durante el MIS4, pueden explicar parcialmente, por qué el momento de máxima extensión de otros glaciares mediterráneos es bastante más antiguo que el máximo glaciar global conocido como LGM, localizado hace $20 \mathrm{ka} \mathrm{BP}$, durante el MIS2. Además de esta asincronía en la extensión máxima de hielo, también existe una discrepancia en el momento de la última deglaciación, que parece haber ocurrido antes en los Pirineos (García-Ruíz et al., 2003; Lewis et al., 2009; Pallàs et al., 2006; González-Sampériz et al., 2005, 2006) y en la Cordillera Cantábrica (Jiménez Sánchez y Farías, 2002), que en otras montañas europeas. Una explicación para este retroceso temprano de los glaciares puede encontrarse en los cambios climáticos abruptos que ocurrieron posteriormente, durante el MIS3.

Como se ha mencionado anteriormente, todavía no tenemos datos palinológicos en Villarquemado que se puedan adscribir con seguridad al MIS4 y que permitan confirmar si el enfriamiento global asociado al inicio del último periodo glacial, fue paralelo a un incremento de la aridez o si, por el contrario, se mantuvieron ciertas condiciones de humedad como se deduce de la geomorfología glaciar. A partir de la sedimentología, observamos cómo a partir de la Unidad V (posible MIS4 según nuestro modelo de edad actual), se produce una paulatina retirada de los humedales y la progradación de los abanicos aluviales distales, indicando una tendencia hacia condiciones más secas, que serán extremas en la siguiente Unidad, la IV. Al igual que parece registrar la secuencia de Villarquemado, la interrupción del crecimiento de espeleotemas en la cercana Cueva del Cristal de Molinos durante el MIS4 (Moreno et al., este volumen), apunta hacia un perio- 
do más bien seco, sugiriendo la existencia de un acusado gradiente de humedad en el NE peninsular durante el comienzo de la última glaciación (Moreno et al., este volumen).

\subsection{MIS3 y MIS2: la variabilidad y el "abrupto" camino hacia el Holoceno}

Desde el trabajo llevado a cabo por Lebreiro et al., (1996), donde las primeras evidencias directas de los denominados Eventos Heinrich (HE) fueron documentadas en sedimentos marinos del litoral portugués, otros registros, principalmente obtenidos de testigos marinos, han destacado abruptas fluctuaciones del clima de la PI durante el MIS3 y el MIS2, sincrónicamente a los ciclos D/O y a los HE (e.g., Cacho et al., 1999; Frigola et al., 2008). Además, los resultados palinológicos obtenidos en los mencionados testigos marinos reflejan una clara respuesta de los ecosistemas terrestres a las fluctuaciones climáticas, registrando un impacto destacable en la cubierta vegetal ante cambios tanto de temperatura como de disponibilidad hídrica (Sánchez-Goñi et al., 2000, 2008; Comborieu Nebout et al., 2002; Roucoux et al., 2005; Fletcher et al., 2010). A diferencia del gran número de registros marinos que cubren el periodo correspondiente al MIS3 y el MIS2, podemos decir que aún son relativamente pocas las secuencias peninsulares que han señalado la respuesta de los sistemas terrestres a las oscilaciones climáticas rápidas de dichos periodos (ver compilaciones en Carrión et al., 2010 y González-Sampériz et al., 2010). De hecho, incluso considerando las secuencias continentales a escala europea, son pocos los registros que han reconstruido detalladamente y con un control cronológico adecuado la dinámica de los ciclos $\mathrm{D} / \mathrm{O}$ (Allen et al., 1999; Wohlfarth et al., 2008). La principal razón para explicar esta ausencia, probablemente se encuentra en que la resolución de análisis generalmente no ha sido suficientemente detallada, limitada en gran parte por la tasa de sedimentación y por la falta de un control cronológico más preciso. Los registros laminados con resolución anual son, probablemente, los mejores candidatos para suplir estas carencias de información, pero hasta el momento no se ha estudiado ninguna secuencia de estas características con la suficiente extensión temporal en la PI.

El registro sedimentario del Cañizar de Villarquemado señala que en la Unidad IV (29-21 m de profundidad) se produjeron niveles del lago reducidos con formación de dolomita, mostrando una tendencia al aumento de la aridez que probablemente comenzó durante el MIS4, y que continúa y se ve incrementada de manera significativa en la parte inferior del MIS3. La alternancia entre depósitos de lago carbonatado y de abanicos aluviales refleja un periodo de inestabilidad hidrológica y de fluctuaciones climáticas, aunque la precisión temporal de los cambios abruptos es imposible con el actual modelo cronológico.

Como se ha mencionado en el apartado anterior de la discusión, apenas existen muestras palinológicas analizadas en la Unidad IV de Villarquemado. En cuanto a la Unidad III, a pesar de presentar aún una caracterización palinológica de baja resolución, podemos observar cómo entre 21 y $18 \mathrm{~m}$ (43-40 ka cal BP según nuestro modelo de edad), se produce un máximo acentuado de AP protagonizado por el pino, que implica el descenso de Artemisia y Chenopodiaceae hasta valores inferiores al 5\%, mientras que entre 18 y $15 \mathrm{~m}$ de profundidad (40-38 ka cal BP), las proporciones se invierten radicalmente, y Juniperus recupera algo de protagonismo, pero con valores que en ningún 
caso vuelven a alcanzar los de la base de la secuencia, ya que apenas llegan a superar el $5 \%$ en estos momentos (Fig. 4). Estos primeros espectros pueden considerarse una aproximación preliminar a la gran variabilidad climática que caracteriza el MIS3.

Por otra parte, dos intervalos palinológicamente estériles según las primeras muestras analizadas quedarían incluidos en la Unidad II: los comprendidos entre 15-13 m, y entre 6-3.5 m de profundidad, lo que cronológicamente coincidiría con el intervalo comprendido entre 38-32 ka cal BP (MIS3) y entre 22-15 ka cal BP (MIS2), respectivamente. Estos periodos coinciden con un nuevo ascenso hacia un máximo en la curva de insolación en el caso del primero (38-32 ka cal BP), y con el LGM y el conocido como “Mistery Interval”-MI el segundo (22-15 ka cal BP). Aun considerando que se está ampliando la resolución de análisis para comprobar la posible esterilidad de estos niveles, no podemos evitar atribuir ciertas condiciones climáticas comunes a ambos eventos. En el caso del intervalo del MIS3, un nuevo ascenso hacia un máximo de insolación de verano, dentro de unas condiciones generales de aridez global, podrían haber provocado un incremento aún mayor de la evapotranspiración debido a las altas temperaturas de verano, produciéndose largos periodos de exposición sub-aérea, la oxidación del sedimento y, por lo tanto, la no preservación del polen. Similares condiciones de aridez se asociarían, tanto al LGM global -que como hemos mencionado anteriormente no se corresponde con el máximo avance de los glaciares en la PI, probablemente porque se trataría de un período más árido en Iberia que en el resto de Europa (Fletcher et al., 2010; González-Sampériz et al., 2010; Moreno et al., 2012), como al MI, ya identificado en numerosas secuencias paleoclimáticas de la PI como un periodo aún más árido que el LGM (Moreno et al., 2010, 2012; Morellón et al., 2010).

En el resto de la Unidad II, la proporción de AP sigue dominada por el pino, que fluctúa entre extremos del 20-80\%, mientras Juniperus y Quercus apenas aparecen a excepción de un pico de más de $10 \%$ de quercíneas alrededor de los $12 \mathrm{~m}$, es decir, en torno a $30 \mathrm{ka}$ cal BP. Chenopodiaceae y Artemisia dibujan una curva algo más constante hasta $\operatorname{los} 7 \mathrm{~m}$, mientras que Poaceae y el componente acuático $(\mathrm{HH})$ presentan valores bajos o incluso inexistentes antes del intervalo de esterilidad polínica mencionado entre 22-15 ka, llegando a superar el 30\% en el caso de las gramíneas, y extremos fuera de la suma polínica que alcanzan el $250 \%$ en el de $\mathrm{HH}$ en el intervalo comprendido entre 14-12 ka cal BP, aproximadamente (Fig. 4).

En el resto de la PI, el registro que proporcionó los primeros indicios sobre las fluctuaciones climáticas del MIS3 fue la secuencia polínica de Banyoles (Pérez-Obiol y Julià, 1994). Estudios posteriores, centrados en la caracterización de facies sedimentológicas e isótopos estables, revelan el impacto de los eventos HE3 y HE2, caracterizados por condiciones áridas y depositadas bajo una reducida lámina de agua (Valero-Garcés et al., 1998; Höbig et al., 2012). Además de Banyoles, otras secuencias localizadas al norte de la PI, como por ejemplo la turbera de El Portalet y el lago Enol, responden también a condiciones áridas y frías durante el HE3 y HE2 (González-Sampériz et al., 2006; Moreno et al., 2010 respectivamente). Particularmente, el registro de El Portalet muestra un incremento claro de los taxones estépicos y la disminución de la frecuencia de Juniperus, al mismo tiempo que se produce un notable aumento de ele- 
mentos siliciclásticos en el sedimento, demostrando que los cambios climáticos abruptos ocurrieron bajo condiciones frías y áridas y tuvieron una respuesta inmediata en la vegetación del entorno (González-Sampériz et al., 2006).

Por último, la parte superior del registro presentado en este trabajo (Unidad I, 3-0.6 m de profundidad), corresponde a facies carbonatadas con intercalaciones de turba que se depositaron desde el comienzo del Holoceno (últimos 11700 años), hasta que la laguna fue desecada. Durante este periodo, la laguna del Cañizar de Villarquemado se expandió y se desarrollaron zonas relativamente profundas, con ecosistemas litorales que sustentaban abundante fauna y flora litoral (gasterópodos, bivalvos, praderas de carófitas), junto a otras zonas de humedales y turberas donde crecía abundante vegetación: un paisaje similar al mosaico de lagos someros y humedales que podemos encontrar en la actualidad en la lagunas de Ruidera, en la cuenca del río Guadiana. Esta variabilidad y mosaico de vegetación acuática se refleja en el registro polínico a partir de las fluctuaciones en la curva de $\mathrm{HH}$, ya que al comienzo de la Unidad I vuelve a descender tras alcanzar valores extremos en el intervalo anterior (final del MIS2). A nivel regional, sin embargo, la vegetación sigue dominada por las herbáceas estépicas y sólo unas pocas coníferas destacan como elementos arbóreos (esencialmente Pinus y Juniperus, aunque este último no aparece en proporciones tan altas como en los metros inferiores de la secuencia). No se registran grandes cambios en la cubierta vegetal hasta bien entrado el Holoceno, cuando la curva de Quercus se desarrolla y llega a dominar el grupo de AP, alcanzando valores de 40 y hasta $60 \%$ en el techo de la secuencia, por primera y única vez en el registro analizado hasta el momento (Fig. 4), mostrando las características típicas de la expansión forestal del interglacial Holoceno en la Iberia Mediterránea (ver compilación en Carrión et al., 2010).

\section{Conclusiones}

Los resultados preliminares obtenidos en la secuencia sedimentaria del Cañizar de Villarquemado indican una alta variabilidad sedimentológica y palinológica a lo largo del último ciclo interglacial-glacial-interglacial (ca. 130000 años de historia ambiental). A grandes rasgos, los datos registrados hasta el momento resultan coherentes con los cambios climáticos a escala milenaria/multimilenaria, aunque presentan ciertas particularidades locales y/o regionales. La mejora del modelo cronológico con la obtención de nuevas dataciones OSL, la culminación del análisis palinológico en los intervalos aún no estudiados, y el aumento de resolución en los que ya poseen cierta caracterización inicial, podrán permitir detectar un mayor detalle de la variabilidad mencionada, identificando también eventos rápidos del tipo D/O o HE.

Por el momento, no podemos precisar si la base del sondeo pertenece cronológicamente al MIS5 ó si incluye también el final del MIS6. Se trata de un periodo húmedo en el que El Cañizar de Villarquemado funcionaría como un mosaico de ambientes de turbera y lago carbonatado, en un entorno en el que Juniperus es el taxón arbóreo más importante registrado. Esto indica un clima de fuerte carácter continental, y resiliencia 
en las formaciones vegetales, alejándose a priori de los espectros polínicos esperables para un interglacial como el Eemiense.

En cuanto al MIS4, y a pesar de las imprecisiones cronológicas, registra una tendencia hacia condiciones más secas con un claro retroceso de los humedales y la progradación de los abanicos aluviales sobre la cuenca de la laguna, pero no poseemos aún información palinológica al respecto. Estos datos, contextualizados regionalmente con registros glaciares y de espeleotemas, apuntan la posible existencia de un gradiente de humedad entre el Pirineo y la Cordillera Ibérica durante el comienzo de la última glaciación.

El momento de mayor aridez de toda la secuencia se corresponde, de acuerdo con los datos disponibles hasta el momento y en particular con las facies sedimentarias, con el MIS3. Los escasos datos palinológicos obtenidos hasta el momento muestran un paisaje desarbolado en el que sólo destacan las proporciones de pino, y una escasa representación de la vegetación hidro-higrófila, confirmando la existencia de condiciones frías/frescas y áridas.

La gran variabilidad climática característica del MIS2 se detecta en la secuencia de Villarquemado por la alternancia de episodios de lago clástico con otros asociados al dominio de abanicos aluviales distales. Palinológicamente, hay que destacar algunos eventos de esterilidad polínica que coinciden con periodos de conocida aridez en Iberia, como el registrado durante el LGM y el MI, lo que podría indicar la existencia de fases prolongadas de exposición sub-aérea y oxidación del contenido esporo-polínico.

Por último, la entrada al Holoceno se registra con un cambio brusco paleohidrológicamente hablando, tal como indican tanto las facies sedimentarias (lago carbonatado con menores ambientes de turbera y humedal asociados), como las variables proporciones de taxones acuáticos. No obstante, la cubierta vegetal general no parece responder a grandes cambios hasta bien entrado el Holoceno, cuando Quercus llega a dominar el estrato arbóreo-arbustivo. Este aparente retraso en la respuesta de la vegetación a los cambios climáticos generales, debe asociarse a la existencia de una importante resiliencia del sistema.

En resumen, las formaciones vegetales del entorno de la laguna del Cañizar de Villarquemado se han visto sometidas a una variabilidad climática de escala milenaria, tal y como apunta la sedimentología, pero matizada por una intensa continentalidad que ha determinado una particular composición del paisaje vegetal, muy adaptado a estas condiciones. Los resultados preliminares presentados en este trabajo confirman el gran potencial que posee esta secuencia, tanto por su extensión temporal como por su particular localización geográfica. La culminación del estudio permitirá completar el complejo escenario paleoclimático que ha caracterizado a la cuenca Mediterránea desde el MIS6 hasta la actualidad.

\section{Agradecimientos}

Este trabajo ha sido financiado gracias a los proyectos nacionales DINAMO (CGL2009-07992), DINAMO2 (CGL2012-33063), LIMNOCLIBER (REN200309130-C02-02), LIMNOCAL (CGL2006-13327-C04-01), GRACCIE-CONSOLIDER 
(CSD2007-00067), CGL2012-38358 y CGL2009-06988/BOS, y al proyecto regional del Gobierno de Aragón-DGA P196/2005. Varios autores (Moreno, Gil-Romera, García-Prieto y Aranbarri) agradecen la financiación recibida de los programas Ramón y Cajal, Juan de la Cierva, Formación de Personal Investigador-FPI y Gobierno Vasco, respectivamente. Además, este trabajo no hubiera sido posible sin la ayuda de la Fundación de la Laguna del Cañizar y su director, José Carlos Rubio, y sin la colaboración de Aida Adsuar, Beatriz Bueno y Raquel López en el procedimiento analítico llevado a cabo en el laboratorio del IPE-CSIC.

\section{Referencias bibliográficas}

AEQUA, Grupo de Trabalho Portugués para o Estudo do Quaternario 1993. El Cuaternario en España y Portugal: Actas de la $2^{a}$ Reunión del Cuaternario Ibérico. IGME, Madrid.

Allen, J.R.M., Huntley, B. 2009. Last Interglacial palaeovegetation, palaeoenvironments and chronology: a new record from Lago Grande di Monticchio, southern Italy. Quaternary Science Reviews 28, 1521-1538.

Allen, J.R.M., Brandt, U., Brauer, A., Hubberten, H.W., Huntley, B., Keller, J. 1999. Rapid environmental changes in southern Europe during the last glacial period. Nature 400 (6746), 740-743.

Allen, J.R.M., Watts, W.A., Huntley, B. 2000. Weichselian palynostratigraphy, palaeovegetation and palaeoenvironment; the record from Lago Grande di Monticchio, southern Italy. Quaternary International 73, 91-110.

Benito, G., Sancho, C., Peña, J.L., Machado, M.J., Rhodes, E.J. 2010. Large-scale karst subsidence and accelerated fluvial aggradation during MIS6 in NE Spain: climatic and paleohydrological implications. Quaternary Science Reviews 29, 2694-2704.

Binka, K., Nitychoruk, J., Dzierzek, J. 2011. Climate stability during the Eemian-new pollen evidence from the Nidzica site, northern Poland. Boreas 40 (2), 342-350.

Blanco Castro, E., Casado, M.A., Costa-Tenorio, M., Escribano, R., García-Antón, M., Génova, M., Gómez Manzaneque, F., Moreno Jarque, J.C., Morla Juaristi, C., Rehato, P., Sáinz Ollero, H. 1997. Los bosques ibéricos. Una interpretación geobotánica. Planeta, Barcelona, 572 pp.

Braconnot, P., Marzin, C., Grégoire, L., Mosquet, E., Martí, O. 2008. Monsoon response to changes in Earth's orbital parameters: comparisons between simulations of the Eemian and of the Holocene. Climate of the Past Discussions 4 (2), 459-493.

Brauer, A., Allen, J.R., Mingram, J., Dulski, P., Wulf, S., Huntley, B. 2007. Evidence for last interglacial chronology and environmental change from Southern Europe. Proceedings of the National Academy of Sciences 104 (2), 450-455.

Broecker, W.S. 2005. Abrupt climate change: causal constraints provided by the paleo-climate record. Earth-Science Reviews 51, 137-54.

Burjachs, F., Julià, R. 1994. Abrupt climatic changes during the last glaciation based on pollen analysis of the Abric Romani, Catalonia, Spain. Quaternary Research 42, 308-315.

Burjachs, F., López-García, J.M., Allué, E., Blain, H.A., Rivals, F., Bennàsar, M. 2012. Palaeoecology of Neanderthals during Dansgaard-Oeschger cycles in northeastern Iberia (Abric Romaní): From regional to global scale. Quaternary International 247, 26-37.

Cacho, I., Grimalt, J., Pelejero, C., Canals, M., Sierro, F.J., Flores, J.A., Shackleton, N. 1999. Dansgaard-Oeschger and Heinrich event imprints in Alboran Sea paleotemperatures. Paleoceanography 14, 698-705.

Carrión, J.S. 1992. Late quaternary pollen sequence from Carihuela Cave, southern Spain. Review of Palaeobotany and Palynology 71, 37-46. 
Carrión, J.S., Fernández, S., González-Sampériz, P., Gil-Romera, G., Badal, E., Carrión-Marco, Y., López-Merino, L., López-Sáez, A., Fierro, E., Burjachs, F. 2010. Expected trends and surprises in the Lateglacial and Holocene vegetation history of the Iberian Peninsula and Balearic Islands. Review of Palaeobotany and Palynology 162, 458-475.

Casas-Sáinz, A.M., De Vicente, G. 2009. On the tectonic origin of Iberian topography. Tectonophysics 474, 214-235.

Chapman, M.R., Shackleton, N.J. 1999. Global ice-volume fluctuations, North Atlantic ice-rafting events, and deep-ocean circulation changes between 130 and $70 \mathrm{ka}$. Geology 27, 795-817.

Combourieu-Nebout, N., Turon, J.L., Zahn, R., Capotondi, L., Londeix, L., Pahnke, K. 2002. Enhanced aridity and atmospheric high-pressure stability over the western Mediterranean during the North Atlantic cold events of the past 50 ky. Geology 30, 863-866.

Dabrio, C.J., Zazo, C., Cabero, A., Goy, J.L., Bardají, T., Hillaire-Marcel, C., González-Delgado, J.A., Lario, J., Silva, P., Borja, F., García-Blázquez, A.M. 2011. Millennial/submillennialscale sea-level fluctuations in western Mediterranean during the second highstand of MIS 5e. Quaternary Science Reviews 30, 335-346.

Dansgaard, W., Johnsen, S.J., Clausen, H.B., Dahl-Jensen, D., Gundestrup, N.S., Hammer, C.U., Hvidberg, C.S., Steffensen, J.P., Sveinbjörnsdóttir, A.E., Jouzel, J., Bond, G. 1993. Evidence for general instability of past climate from a 250-kyr ice-core record. Nature 364, 218-220.

De Beaulieu, J.L., Reille, M. 1992a. Long Pleistocene pollen sequences from the Velay Plateau (Massif Central, France). Vegetation History and Archaeobotany 1, 233-242.

De Beaulieu, J.L., Reille, M. 1992b. The last climatic cycle at La Grande Pile (Vosges, France), a new pollen profile. Quaternary Science Reviews 11 (4), 431-438.

Del Valle, J. 1993. El régimen pluviométrico medio de la cuenca del río Jiloca. Geographicalia 30, 145-154.

Domínguez-Villar, D., Vázquez-Navarro, J.A., Cheng, H., Edwards, R.L. 2011. Freshwater tufa record from Spain supports evidence for the past interglacial being wetter than the Holocene in the Mediterranean region. Global and Planetary Change 77, 129-141.

Drescher-Schneider, R. 2000. The Riss-Wurm interglacial from west to east in the Alps: an overview of the vegetational succession and climatic development. Geologie en Mijnbouw 79, 233-240.

Fernández, S., Fuentes, N., Carrión, J.S., González-Sampériz, P., Montoya, E., Gil, G., Vega-Toscano, G., Riquelme, J.A. 2007. The Holocene and Upper Pleistocene pollen sequence of Carihuela cave, southern Spain. Geobios 40, 75-90.

Fletcher, W.J., Sánchez-Goñi, M.F., Allen, J.R.M., Cheddadi, R., Combourieu-Nebout, N., Huntley, B., Lawson, I., Londeix, L., Magri, D., Margari, V., Muller, U.C., Naughton, F., Novenko, E., Roucoux, K., Tzedakis, P.C. 2010. Millennial-scale variability during the last glacial in vegetation records from Europe. Quaternary Science Reviews 29 (21), 2839-2864.

Follieri, M., Magri, D., Sadori, L. 1988. A 250,000-year pollen record from Valle di Castiglione (Roma). Pollen et Spores 30, 329-356.

Forsström, L. 2001. Duration of interglacials: a controversial question. Quaternary Science Reviews 20 (15), 1577-1586.

Frigola, J., Moreno, A., Cacho, I., Canals, M., Sierro, F.J., Flores, J.A., Grimalt, J. 2008. Evidence of abrupt changes in Western Mediterranean Deep Water circulation during the last $50 \mathrm{kyr}$ : A high-resolution marine record from the Balearic Sea. Quaternary International 181 (1), 88-104.

Ganopolski, A., Robinson, A. 2011. Palaeoclimate: The past is not the future. Nature Geoscience 4, 661-663.

García-Ruiz, J.M., Valero-Garcés, B.L., Martí-Bono, C., González-Sampériz, P. 2003. Asynchroneity of maximum glacier advances in the central Spanish Pyrenees. Journal of Quaternary Sciences 18, 61-72. 
García-Ruiz, J.M., Moreno, A., González-Sampériz, P., Valero-Garcés, B.L., Martí-Bono, C. 2010. La cronología del último ciclo glaciar en las montañas del sur de Europa: una revisión. Cuaternario y Geomorfología 24, 35-46.

García-Ruiz, J.M., Martí-Bono, C., Peña-Monné, J.L., Sancho, C., Rhodes, E.J., Valero-Garcés, B., González-Sampériz, P., Moreno, A. 2012. Glacial and fluvial deposits in the Aragón Valley, Central-Western Pyrenees: Chronology of the Pyrenean Late Pleistocene glaciers. Geografiska Annaler, Series A, Physical Geography 95, 15-32.

Gómez-Orellana, L., Ramil-Rego, P., Muñoz-Sobrino, C. 2007. The Würm in NW Iberia: a pollen record from area Longa (Galicia). Quaternary Research 67, 438-452.

González-Sampériz, P., Valero-Garcés, B.L., Carrión, J.S., Peña-Monné, J.L., García-Ruiz, J.M., Martí-Bono, C. 2005. Glacial and Lateglacial vegetation in northeastern Spain: new data and a review. Quaternary International 140, 4-20.

González-Sampériz, P., Valero-Garcés, B.L., Moreno, A., Jalut, G., García-Ruiz, J.M., Martí-Bono, C., Delgado-Huertas, A., Navas, A., Otto, T., Dedoubat, J.J. 2006. Climate variability in the Spanish Pyrenees during the last 30,000 yr revealed by the El Portalet sequence. Quaternary Research 66, 38-52.

González-Sampériz, P., Leroy, S.A.G., Carrión, J.S., Fernández, S., García-Antón, M., Gil-García, M.J., Uzquiano, P., Valero-Garcés, B.L., Figueiral, I. 2010. Steppes, savannahs, forests and phytodiversity reservoirs during the Pleistocene in the Iberian Peninsula. Review of Palaeobotany and Palynology 162, 427-457.

Gracia, F.J., Gutiérrez, F., Gutiérrez, M. 2003. The Jiloca karst polje-tectonic graben (Iberian Range, NE Spain). Geomorphology 52 (3-4), 215-231.

Guan, Q., Pan, B., Gao, H., Li, B., Wang, J., Su, H. 2007. Instability characteristics of the East Asian Monsoon recorded by high-resolution loess sections from the last interglacial (MIS5). Scientific China Series D 7, 1067-1075.

Guiot, J., Cheddadi, R. 2004. Variabilité des écosystèmes terrestres et du climat sur un cycle glaciaire-interglaciaire. Comptes Rendus Geoscience 336, 667-675.

Guiot, J., Pons, A., De Beaulieu, J.L. 1989. A 140,000-year continental climate reconstruction from two European pollen records. Nature 338, 309-313.

Gutiérrez, F., Gracia, F.J. 1997. Palaeosurfaces: Recognition, Reconstruction and Palaeoenvironmental Interpretation. Geological Society Spec. 120, 147-158.

Gutiérrez, F., Gutiérrez, M., Gracia, F.J., McCalpin, J.P., Lucha, P., Guerrero, J. 2008. Plio-Quaternary extensional seismotectonics and drainage network development in the central sector of the Iberian Chain (NE Spain). Geomorphology 102, 21-42.

Gutiérrez, F., Gracia, F.J., Gutiérrez, M., Lucha, P., Guerrero, J., Carbonel, D. 2012. A review on Quaternary tectonic and nontectonic faults in the central sector of the Iberian Chain, NE Spain. Journal of Iberian Geology 38, 145-160.

Heegaard, E., Birks, H.J.B., Telford, R.J. 2005. Relationships between calibrated ages and depth in stratigraphical sequences: an estimation procedure by mixed-effect regression. The Holocene 15, 612-618.

Höbig, N., Weber, M.E., Kehl, M., Weniger, G.C., Julià, R., Melles, M., Fülöp, R.K., Vogel, H., Reicherter, K. 2012. Lake Banyoles (northeastern Spain): A Last Glacial to Holocene multiproxy study with regard to environmental variability and human occupation. Quaternary International 274, 205-218.

Hodge, E.J., Richards, D.A., Smart, P.L., Andreo, B., Hoffmann, D.L., Mattey, D.P. 2008. Effective precipitation in southern Spain $(\sim 266$ to $46 \mathrm{ka})$ based on a speleothem stable carbon isotope record. Quaternary Research 69, 447-457.

Hughes, P.D., Woodward, J.C. 2008. Timing of glaciation in the Mediterranean mountains during the last cold stage. Journal of Quaternary Science 23, 575-588. 
IPCC 2007. IPCC Fourth Assessment Report: Climate Change 2007 (AR4).

Jiménez-Sánchez, M., Farias, P. 2002. New radiometric and geomorphologic evidences of a last glacial maximum older than $18 \mathrm{ka}$ in SW European mountains: the example of Redes Natural Park (Cantabrian Mountains, NW Spain). Geodinamica Acta 15, 93-101.

Klotz, S., Guiot, J., Mosbrugger, V. 2003. Continental European Eemian and early Würmian climate evolution: comparing signals using different quantitative reconstruction approaches based on pollen. Global and Planetary Change 36 (4), 277-294.

Kukla, G., McManus, J.F., Rousseau, D.D., Chuine, I. 1997. How long and how stable was the last interglacial? Quaternary Science Reviews 16, 605-612.

Lafuente, P. 2011. Tectónica activa y paleosismicidad de la falla de Concud (Cordillera Ibérica central). Tesis Doctoral, Universidad de Zaragoza, Zaragoza, 338 pp.

Lafuente, P., Arlegui, L.E., Liesa, C.L., Simón, J.L. 2011 . Paleoseismological analysis of an intraplate extensional structure: the Concud fault (Iberian Chain, eastern Spain). International Journal of Earth Sciences 100, 1713-1732.

Laskar, J., Robutel, P., Joutel, F., Gastineau, M., Correia, A.C.M., Levrard, B. 2004 . A long-term numerical solution for the insolation quantities of the Earth. Astronomy \& Astrophysics 428 , 261-285.

Lebreiro, S.M., Moreno, J.C., McCave, I.N., Weaver, P.P.E. 1999. Evidence for Heinrich layers off Portugal (Tore Seamount: $39^{\circ} \mathrm{N}, 12^{\circ} \mathrm{W}$ ). Marine Geology 131, 47-56.

Leemans, R., Redman, C., Schimel, D. 2007. Sustainability or collapse: what can we learn from integrating the history of humans and the rest of nature? Ambio 36, 522-527.

Lewis, C.J., McDonald, E.V., Sancho, C., Peña, J.L., Rhodes, E.J. 2009. Climatic implications of correlated Upper Pleistocene glacial and fluvial deposits on the Cinca and Gállego Rivers (NE Spain) based on OSL dating and soil stratigraphy. Global and Planetary Change 67, 141-152.

Lézine, A.M., Von Grafenstein, U., Andersen, N., Belmecheri, S., Bordon, A., Caron, B., Cazet, J.P., Erlenkeuser, H., Fouache, E., Grenier, C., Huntsman-Mapila, P., Hureau-Mazaudier, D., Manelli, D., Mazaud, A., Robert, C., Sulpizio, R., Tiercelin, J.J., Zanchetta, G., Zeqollari, Z. 2010. Lake Ohrid, Albania, provides an exceptional multi-proxy record of environmental changes during the last glacial-interglacial cycle. Palaeogeography, Palaeoclimatology, Palaeoecology 287, 116-127.

López-Martín, F., Cabrera Millet, M., Cuadrat, J.M. (eds.) 2007. Atlas climático de Aragón. Gobierno de Aragón, Servicio de Información y Educación Ambiental, Zaragoza, 222 pp.

Magri, D., Tzedakis, P.C. 2000. Orbital signatures and long-term vegetation patterns in the Mediterranean. Quaternary International 73, 69-78.

Montero de Burgos, J.L., González Rebollar, J.L. 1983. Diagramas bioclimáticos. ICONA, Madrid. Moore, P.D., Webb, J.A., Collison, M.E. 1991. Pollen analysis. Blackwell Scientific, Oxford, 216 pp. Morellón, M., Valero-Garcés, B.L., Vegas-Villarrúbia, T., González-Sampériz, P., Romero, Ó., Delgado-Huertas, A., Mata, P., Moreno, A., Rico, M., Corella, J.P. 2009. Lateglacial and Holocene palaeohydrology in the western Mediterranean region: the Lake Estanya record (NE Spain). Quaternary Science Reviews 28, 2582-2599.

Moreno, A., González-Sampériz, P., Morellón, M., Valero-Garcés, B.L., Fletcher, W.J. 2010. Northern Iberian abrupt climate change dynamics during the last glacial cycle: A view from lacustrine sediments. Quaternary Science Reviews 36, 139-153.

Müller, U.C. 2000. A Late-Pleistocene pollen sequence from the Jammertal, south-western Germany with particular reference to location and altitude as factors determining Eemian forest composition. Vegetation History and Archaeobotany 9, 125-131. 
Müller, U.C., Jo, P., Bibus, E. 2003. Vegetation response to rapid climate change in Central Europe during the past 140,000 yr based on evidence from the Furamoos pollen record. Quaternary Research 59, 235-245.

Nikolova, I., Yin, Q., Berger, A., Singh, U.K., Karami, M.P. 2012. The last interglacial (Eemian) climate simulated by LOVECLIM and CCSM3. Climate of the Past Discussions 8, 5293-5340.

Pallàs, R., Rodés, Á., Braucher, R., Carcaillet, J., Ortuño, M., Bordonau, J., Bourle, D., Vilaplana, J.M., Masana, E., Santanach, P. 2006. Late Pleistocene and Holocene glaciation in the Pyrenees: a critical review and new evidence from 10Be exposure ages, south-central Pyrenees. Quaternary Science Reviews 25, 2937-2963.

Peña Monné, J.L., Sancho, C., Lewis, C., McDonald, E., Rhodes, E. 2004. Datos cronológicos de las morrenas terminales del glaciar del Gállego y su relación con las terrazas fluvioglaciares (Pirineo de Huesca). En Geografía Física de Aragón, aspectos generales y temáticos, J.L. Peña Monné, L.A. Longares, M. Sánchez (eds.), Universidad de Zaragoza e Institución Fernando el Católico, Zaragoza, pp. 71-84.

Pérez-Obiol, R., Julià, R. 1994. Climatic change on the Iberian Peninsula recorded in a 30,000-yr pollen record from Lake Banyoles. Quaternary Research 41, 91-98.

Pini, R., Ravazzi, C., Donegana, M. 2009. Pollen stratigraphy, vegetation and climate history of the last $215 \mathrm{ka}$ in the Azzano Decimo core (plain of Friuli, north-eastern Italy). Quaternary Science Reviews 28, 1268-1290.

Pons, A., Reille, M. 1988. The Holocene-and Upper Pleistocene pollen record from Padul (Granada, Spain): a new study. Palaeogeography, Palaeoclimatology, Palaeoecology 66, 243-249.

Pons, A., Guiot, J., De Beaulieu, J.L., Reille, M. 1992. Recent contributions to the climatology of the last glacial-interglacial cycle based on French pollen sequences. Quaternary Science Reviews 11, 439-448.

Reimer, P.J., Baillie, M.G.L., Bard, E., Bayliss, A., Beck, J.W., Blackwell, P.G., Bronk Ramsey, C., Buck, C., Burr, G., Edwards, R., Friedrich, M., Grootes, P., Guilderson, T., Hajdas, I., Heaton, T., Hogg, A., Hughen, K., Kaiser, K., Kromer, B., McCormac, F., Manning, S., Reimer, R., Richards, D., Southon, J., Talamo, S., Turney, C., Van der Plicht, J., Weyhenmeier, C. 2009. IntCa109 and Marine09 radiocarbon age calibration curves, 0-50.000 years cal BP. Radiocarbon 51, 1111-1150.

Roucoux, K.H., Da Abreu, L., Shackleton, N.J., Tzedakis, P.C. 2005. The response of NW Iberian vegetation to North Atlantic climate oscillations during the last 65 kyr. Quaternary Science Reviews 24, 1637-1653.

Rubio, J.C. 2004. Contexto hidrogeológico e histórico de los humedales del Cañizar. Consejo de Protección de la Naturaleza de Aragón, Serie investigación, Zaragoza.

Sánchez-Goñi, M.F., Eynaud, F., Turon, J.L., Shackleton, N.J. 1999. High resolution palynological record off the Iberian margin: direct land-sea correlation for the Last Interglacial complex. Earth and Planetary Science Letters 171, 123-137.

Sánchez-Goñi, M.F.S., Turon, J.L., Eunaud, F., Gendreau, S. 2000. European climatic response to millennial-scale changes in the atmosphere-ocean system during the Last Glacial period. Quaternary Research 54 (3), 394-403.

Sánchez-Goñi, M.F., Landais, A., Fletcher, W.J., Naughton, F., Desprat, S., Duprat, J. 2008. Contrasting impacts of Dansgaard-Oeschger events over a western European latitudinal transect modulated by orbital parameters. Quaternary Science Reviews 27, 1136-1151.

Satkunas, J., Grigiene, A., Velichkevich, F., Robertsson, A., Sandgren, P. 2003. Upper Pleistocene stratigraphy at the Medininkai site, eastern Lithuania: a continuous record of the EemianWeichselian sequence. Boreas 32, 627-641.

Schnurrenberger, D., Russell, J., Kelts, K. 2003. Classification of lacustrine sediments based on sedimentary components. Journal of Paleolimnology 29, 141-154. 
Shackleton, N.J. 1969. The last interglacial in the marine and terrestrial records. Proceedings of the Royal Society of London. Series B. Biological Sciences 174, 135-154.

Sier, M.J., Roebroeks, W., Bakels, C., Dekkers, M., Brühl, E., De Loecker, D., Gaudzinski-Windheuser, S., Hesse, N., Jagich, A., Kindler, L., Kujper, W., Laurat, T., Mücher, H., Penkman, K., Richter, D., Van Hisbergen, D. 2011. Direct terrestrial marine correlation demonstrates surprisingly late onset of the last interglacial in central Europe. Quaternary Research 75, 213-218.

Simón, J.L. 1983. Tectónica y neotectónica del sistema de fosas de Teruel. Teruel 69, 21-97.

Simón, J.L. 1989. Late Cenozoic stress field and fracturing in the Iberian Chain and Ebro Basin (Spain). Journal of Structural Geology 11, 285-294.

Simón, J.L., Rubio, J.C., Soriano, M.A. 2010. Sobre el origen y edad de la depresión del Jiloca (Teruel, Cordillera Ibérica centro-oriental). Geogaceta 48, 183-186.

Solomon, S., Qin, D., Manning, M., Marquis, M., Averyt, K., Tignor, M.M.B. 2007. Climate Change 2007: The Physical Science basis: Summary for policymakers. Contribution of Working Group I to the Fourth Assessment Report of the Intergovernmental Panel on Climate Change IPCC. Cambridge University Press, Cambridge.

Stuiver, M., Reimer, P.J. 1993. Radiocarbon calibration program, revision 3.0. Radiocarbon 35, 215-230.

Tzedakis, P.C. 1994. Vegetation change through glacial-interglacial cycles: a long pollen sequence perspective. Philosophical Transactions of the Royal Society of London. Series B: Biological Sciences 345, 403-432.

Tzedakis, P.C. 2003. Timing and duration of Last Interglacial conditions in Europe: a chronicle of a changing chronology. Quaternary Science Reviews 22, 763-768.

Tzedakis, P.C., Frogley, M.R., Heaton, T.H.E. 2003. Last Interglacial conditions in southern Europe: evidence from Ioannina, northwest Greece. Global and Planetary Change 36, 157-170.

Valero-Garcés, B.L., Zeroual, E., Kelts, K. 1998. Arid phases in the western Mediterranean region during the Last Glacial Cycle reconstructed from lacustrine records. Paleohydrology and Environmental Change, pp. 67-80.

Van Kolfschoten, T.H., Gibbard, P.L., Knudsenc, K.L. 2003. The Eemian Interglacial: a Global Perspective. Introduction. Global and Planetary Change 36, 147-149.

Vogel, H., Wagner, B., Zanchetta, G., Sulpizio, R., Rosén, P. 2010. A paleoclimate record with tephrochronological age control for the last glacial-interglacial cycle from Lake Ohrid, Albania and Macedonia. Journal of Paleolimnology 44, 295-310.

Waelbroeck, C., Labeyrie, L., Michel, E., Duplessy, J.C., McManus, J.F., Lambeck, K. 2002. Sealevel and deep water temperature changes derived from benthic foraminifera isotopic records. Quaternary Science Reviews 21, 295-305.

Wohlfarth, B., Veres, D., Aampel, L., Lacourse, T., Blaauw. M., Preusser, F. 2008. Rapid ecosystem response to abrupt climate changes during the last glacial period in western Europe, 40-16 ka. Geology 36, 407-410.

Woillard, G.M., Mook, W.G. 1982. Carbon-14 dates at Grande Pile: correlation of land and sea chronologies. Science 215, 159-161.

Wolff, E.W., Chappellaz, J., Blunier, T., Rasmussen, S.O., Svensson, A. 2010. Millennial-scale variability during the last glacial: the ice core record. Quaternary Science Reviews 29, 2828-2838. 